\title{
Turbulent Kinetic Energy Production in the Vane of a Low-Pressure Linear Turbine Cascade with Incoming Wakes
}

\author{
V. Michelassi and J. G. Wissink \\ Institut für Hydromechanik, Universität Karlsruhe, Kaiserstrasse 12, 76128 Karlsruhe, Germany \\ Correspondence should be addressed to V. Michelassi; vittorio.michelassi@gmail.com
}

Received 5 November 2014; Accepted 4 January 2015

Academic Editor: Funazaki Ken-ichi

Copyright ( 2015 V. Michelassi and J. G. Wissink. This is an open access article distributed under the Creative Commons Attribution License, which permits unrestricted use, distribution, and reproduction in any medium, provided the original work is properly cited.

\begin{abstract}
Incompressible large eddy simulation and direct numerical simulation of a low-pressure turbine at $\operatorname{Re}=5.18 \times 10^{4}$ and $1.48 \times 10^{5}$ with discrete incoming wakes are analyzed to identify the turbulent kinetic energy generation mechanism outside of the blade boundary layer. The results highlight the growth of turbulent kinetic energy at the bow apex of the wake and correlate it to the stress-strain tensors relative orientation. The production rate is analytically split according to the principal axes, and then terms are computed by using the simulation results. The analysis of the turbulent kinetic energy is followed both along the discrete incoming wakes and in the stationary frame of reference. Both direct numerical and large eddy simulation concur in identifying the same production mechanism that is driven by both a growth of strain rate in the wake, first, followed by the growth of turbulent shear stress after. The peak of turbulent kinetic energy diffuses and can eventually reach the suction side boundary layer for the largest Reynolds number investigated here with higher incidence angle. As a consequence, the local turbulence intensity outside the boundary layer can grow significantly above the free-stream level with a potential impact on the suction side boundary layer transition mechanism.
\end{abstract}

\section{Introduction}

Typically, the time-averaged flow field of undisturbed plane wakes consists of two equally strong, slowly diverging vortex sheets of opposite orientation. The rate at which the sheets diverge is proportional to $d^{1 / 2}$, where $d$ is the distance to the origin of the wake (see, e.g., Schlichting [1]), indicating that for large $d$ the two vortex sheets will be roughly parallel. Each vortex sheet corresponds to a plane shear layer not unlike a flat plate boundary layer. In a turbulent plane wake, each shear layer is associated with a peak in the turbulent kinetic energy, $k$, indicating that the production of $k$ is concentrated near the regions of high shear.

Castro and Bradshaw [2] and Gibson and Rodi [3] analysed the flow and turbulence structure of a highly curved mixing layer. The mixing layer under investigation bounds a normally impinging plane irrotational jet. In the experiment, turbulence was discovered to be first attenuated. Then, approximately after the first $50-60 \%$ of the curve, the normal and shear stresses were first amplified and exceeded the plane-layer values reached in relaxation region further downstream when the flow curvature vanishes. The analysis indicated that the overshoot of turbulent quantities was mostly due to shear-stress production.

In the absence of strain, Moser et al. [4] observed a selfsimilar evolution of plane wakes. The effect of the presence of uniform mean strain was studied by Rogers [5], who performed direct numerical simulations of turbulent, timeevolving strained wakes using a pseudo-spectral method. In all his simulations, the strain was applied to the same self-similar wake flow field. He found that though the main flow reacts quickly on the applied strain, the response of turbulence to strain is slower; changes in the turbulence intensity could not keep pace with changes in the mean wake velocity. Turbulence is produced by two competing mechanisms: shear and strain. Rogers found that, when the direction of compression is parallel to the centre-line of the wake (case C), the wake-width grows exponentially as the wake velocity deficit increases. The normal Reynolds stresses, $u^{\prime} u^{\prime}, v^{\prime} v^{\prime}, w^{\prime} w^{\prime}$, and also $u^{\prime} v^{\prime}$ are all found to increase in 
TABLE 1: Details of the blade geometry and conditions.

\begin{tabular}{lccccccc}
\hline Test & Re & $\gamma$-deg & $\beta_{1}$-deg & $\beta_{2}$-deg & $t_{b} / t$ & $U_{\text {bar }}$ & $d_{b} / C$ \\
\hline $\mathrm{L}$ & $5.18 \times 10^{4}$ & 30.72 & 45.5 & 63.2 & 0.5 & -0.41 \\
$\mathrm{H}$ & $1.48 \times 10^{5}$ & 30.72 & 37.7 & 63.2 & 1.0 & -1.204 & 0.02 \\
\hline
\end{tabular}

time while the typical structure of the time-averaged wake, that is, the two more or less parallel shear layers, remains intact. When the direction of expansion is parallel to the centre-line of the wake (case D), the wake-width, the wake velocity deficit, and the Reynolds stresses all decrease in time, eventually degrading the structure of the wake.

In summary, the measurements by Castro and Bradshaw [2] allow studying the evolution of shear layers (i.e. half portion of a wake) in presence of strong flow-core turning, whereas the DNS by Rogers [5] focus on the effect of strain on planar straight wakes. Both are relevant to the flow in a turbomachine in which the wakes produced by the preceding blade row are periodically ingested into a blade vane. In particular, while a plane wake travels through the passage between two turbine blades it is severely strained and distorted by the main flow. In contrast to the study of Rogers, the actual direction of the mean strain relative to the centreline of the wake varies with the actual location. Moreover, the direction of shear, individuated by the wakes, differs from the flow direction because of the relative motion between blades and wakes. Hence, differences arise with respect to the flow geometry by Castro and Bradshaw too, in which the direction of shear is aligned with the core flow.

Wu and Durbin [6] performed the DNS of the flow in a low-pressure (LP) linear turbine rotor blade with periodic incoming wakes. The wakes are subject to both flow curvature (as in Castro and Bradshaw [2]) and strain (as in Rogers [5]). The simulations revealed a peak in the turbulent kinetic energy located near the bow-apex of the wake, where the direction of compression is aligned with the centre-line of the wake, corresponding to case $\mathrm{C}$ of Rogers. Near the pressure side $\mathrm{Wu}$ and Durbin observed that the direction of expansion was almost aligned with the centre-line of the wake, corresponding to case $\mathrm{D}$ of Rogers. In the present paper we aim to further analyse the production of turbulence in the plane turbulent wake as it travels through the free-stream region of a turbine cascade.

\section{Description of the Test Cases}

The geometry under investigation is that of the low-pressure aft-loaded turbine blade T106. The flow around this blade assembled in a linear cascade was measured by Stadtmüller [7] and by Stadtmüller and Fottner [8]. In the experiments the blade aspect ratio (h/c) is 1.76 , and the blade chord is $100 \mathrm{~mm}$. Therefore the flow at mid-span can be considered nearly two-dimensional and the three-dimensional computer simulations can be performed under the assumption of a homogeneous flow in the span-wise direction. The measurements were carried out at $\operatorname{Re}=5.18 \times 10^{4}$ and $2 \times 10^{5}$ (based on inlet conditions and axial chord) with the effect of an upstream row of blades simulated by a moving bar wake

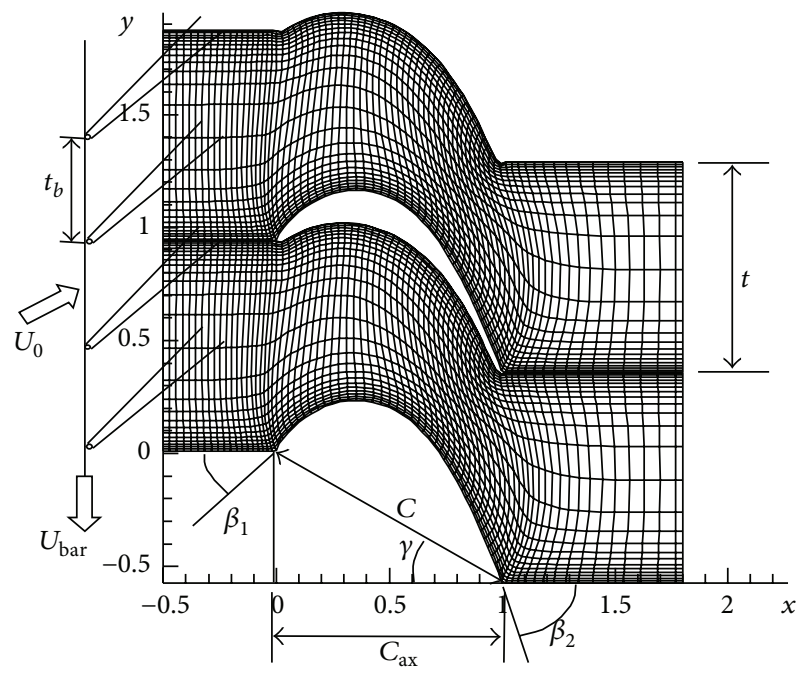

FIGURE 1: Flow configuration and grid for LES at Re $=5.18 \times 10^{4}$ (every 6th grid line is shown in both stream-wise and pitch-wise directions).

generator. The bar diameter to pitch ratio is $d_{b} / C=0.02$. Unfortunately, for the larger Re only, the bar-to-blade pitch ratio is not an integer number $(0.4 / 0.525)$. To yield an exactly periodic flow, the simulations should hence be carried out by using eight blade vanes, which would require an excessive computational effort. Therefore we will focus on the DNS data by Wissink [9] and the LES data by Michelassi et al. [10], which refer both to the flow at the lower Reynolds number with a wake-to-blade pitch ratio of 0.5 (see test L in Table 1). The same geometry was also selected by Wu and Durbin [6] for a DNS at the significantly larger Re of $1.48 \times 10^{5}$ with a wake-to-blade pitch ratio of one. This DNS was used as a reference data set by Michelassi et al. [10] who performed the LES of the same flow. The latter data set will also be used for further analysis under different operating conditions with respect to $[9,10]$ (see test $\mathrm{H}$ in Table 1 ).

\section{Computational Details}

3.1. Computational Grids. The blade stagger angle $\gamma$, the inlet flow angle $\beta_{1}$, and the outlet flow angle $\beta_{2}$, defined with respect to the axial direction as displayed in Figure 1, are summarised in Table 1. The grid employed in both the DNS and LES was carefully selected using the elliptic grid generation algorithm proposed by Hsu and Lee [11]. This elliptic mesh generation ensured a nearly orthogonal grid close to the blade walls and it was therefore adopted for all the simulations. Table 2 summarises the adopted grid sizes. The DNS grid [9] (see run D) consists of $1014 \times 266$ $\times 64$ nodes in the stream-wise, pitch-wise, and span-wise 
TABLE 2: Summary of the test runs.

\begin{tabular}{lcccc}
\hline Run & Test & Grid & $h$ & SGS model \\
\hline D & L & $1014 \times 260 \times 64$ & $0.2 \times C_{\mathrm{ax}}$ & None \\
L1 & L & $454 \times 144 \times 32$ & $0.15 \times C_{\mathrm{ax}}$ & Dyn. Smagorinsky \\
L2 & H & $646 \times 256 \times 64$ & $0.15 \times C_{\mathrm{ax}}$ & Dyn. Smagorinsky \\
\hline
\end{tabular}

directions, respectively. The grid extension in the span-wise direction and downstream of the trailing edge is $0.20 C_{\mathrm{ax}}$ and $1.0 C_{a x}$, respectively. The grid of the LES under the same operating conditions (see run L1) [10] consists of $448 \times 144$ $\times 32$ points in the stream-wise, pitch-wise, and span-wise directions, respectively, and provides a fair representation of the DNS flow field [10]. The LES grid extension in the spanwise direction and downstream of the trailing edge is $0.15 C_{\mathrm{ax}}$ and $0.8 C_{\mathrm{ax}}$, respectively. Further details on the grid resolution are available in $[9,10]$. The LES at $R e=1.48 \times 10^{5}$ adopts $646 \times$ $256 \times 64$ nodes in the stream-wise, pitch-wise, and span-wise directions, respectively. As in the DNS by Wu and Durbin [6], the grid extends $0.5 \times C_{\mathrm{ax}}$ upstream of the leading edge of the blades and $1.0 \times C_{\mathrm{ax}}$ downstream of the trailing edge. The span-wise width is $h=0.15 \times C_{\mathrm{ax}}$. The grid in the span-wise direction is uniform for all runs.

3.2. Boundary Conditions and Phase Averaging. A no-slip boundary condition is enforced at the surface of the blade and a periodic flow condition is enforced in the pitch-wise direction. The latter condition is not critical since the size of the expected flow structures is a small percentage of the blade pitch. Since the dynamically relevant motions are expected to have a span-wise extent smaller than the size of the computational domain, it is possible to enforce the periodicity of the instantaneous flow in the span-wise direction too. The respective values of the span-wise extension of the computational domain, H (see Table 2), had been carefully selected to avoid undesired effects on the development of flow structures. The inflow boundary condition for the wakes is enforced by using the database made available by $\mathrm{Wu}$ and Durbin, who generated the incoming turbulent wakes-like data with preliminary LES [12]. In all runs the wake data closely resembles those adopted in the DNS [6] in which the wake half-width is $0.04 \times C_{a x}$ and the maximum mean wake velocity deficit is $18 \%$.

Apart from the Reynolds number and the inlet flow angle, the two test cases $\mathrm{L}$ and $\mathrm{H}$ differ in the incoming wake-toblade pitch ratio and wake tangential speed. In particular, in test case $\mathrm{H}$ the tangential speed of the wake is significantly larger than in case L. In order to properly resolve the wake in both space and time, one period $T=t_{b} / U_{b}$ was resolved using 9600 time steps in run D, 4800 time steps in run L1, and 10240 in run L2. In all runs, this choice implies a maximum CFL number of approximately $0.30-0.50$. In all the simulations the flow was allowed to develop for five periods $T$, that is, five wake passes. After this start-up period the flow developed a periodic behaviour and it was possible to initiate the phase averaging of the flow field. Phase-averaging is performed over ten further periods. Run D stored 240 phases, while this number is reduced to 120 and 64 for runs L1 and L2, respectively. In the following, $\Phi$, with $0 \leq \Phi<1$, corresponds to the phase of a phase-averaged flow field. Angular brackets denote phase-averaged quantities.

3.3. Calculation Method and Test Runs. The DNS and LES of the flow around the T106 turbine blade have been performed using the LESOCC code [13]. The incompressible continuity and momentum equations are discretized by means of a cellcentred finite volume method. Mass conservation is ensured by the implicit solution of a Poisson equation for the pressure correction, which is complemented with a Fourier solver in the span-wise direction that substantially reduces the computational effort. The equations are solved by marching in time with a three-stage Runge-Kutta algorithm. The mass conservation step was converged to a residual of $10^{-8}$. The SGS model used in the LES is the dynamic model by Germano et al. [14] with the modification of Lilly [15]. It employs an eddy viscosity model together with a procedure of reducing the model constant whenever the flow is well resolved. For the present computation, the SGS model employs filtering and averaging in the homogeneous span-wise direction. The test runs, which are summarised in Table 2, refer to two different Reynolds numbers and incoming wake frequencies. Therefore, the relatively large data availability allows the flow inside the blade vane to be analysed in different configurations and with different modelling assumptions. The next sections will deal with the DNS at $\mathrm{Re}=5.18 \times 10^{4}$ and the LES at both Re $=5.18 \times 10^{4}$ and $\operatorname{Re}=1.48 \times 10^{5}$.

\section{Flow Visualisation}

The dynamics of the wake, while it is convected inside the blade vane, has already been extensively discussed by Wu and Durbin [6] for the $\operatorname{Re}=1.48 \times 10^{5}$ case and by Wissink [9] as well as by Michelassi et al. [10] for the $\mathrm{Re}=5.18 \times 10^{4}$ case, both in terms of instantaneous and phase-averaged velocities, and it will not be repeated here. In particular, both Wu and Durbin [6] and Wissink [9] observed an accumulation of turbulent kinetic energy along the wake path inside the turbine vane. This phenomenon is illustrated in the plots of the phaseaveraged turbulent kinetic energy, $\langle k\rangle$, of Figure 2. The phaseaveraged three-dimensional flow field around the T106 blade is first averaged in the span-wise homogeneous direction. This allows extracting the two-dimensional phase-averaged snapshots of the turbulence quantities. The plots refer to two different phases for both the LES and the DNS at Re $=5.18$ $\times 10^{4}$ and the LES at $\operatorname{Re}=1.48 \times 10^{5}$. In a DNS, basically all velocity fluctuations are resolved and thus contribute to the turbulent kinetic energy. In the LES, only the resolved velocity fluctuations are used to compute the turbulent kinetic energy. The absence of the SGS model contribution explains the slightly lower overall values of $\langle k\rangle$ shown by LES with respect to DNS. In all simulations, at the inlet the wake is the only source of velocity fluctuations in an otherwise uniform flow field. This is the reason for the absence of any visible turbulent kinetic energy in between wakes in the first $40-50 \%$ of the computational domain. Hence, the wake is easily identified with the flow regions in which the turbulent kinetic energy departs from zero. The figures illustrate how the turbulent 


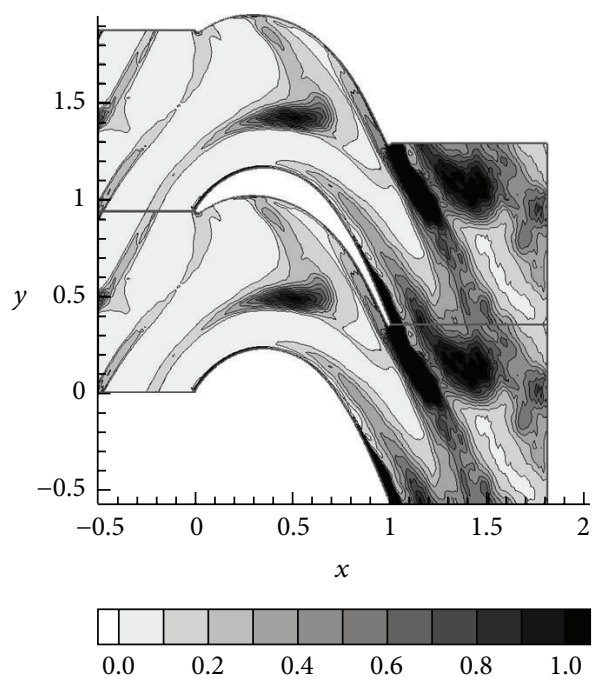

(a)
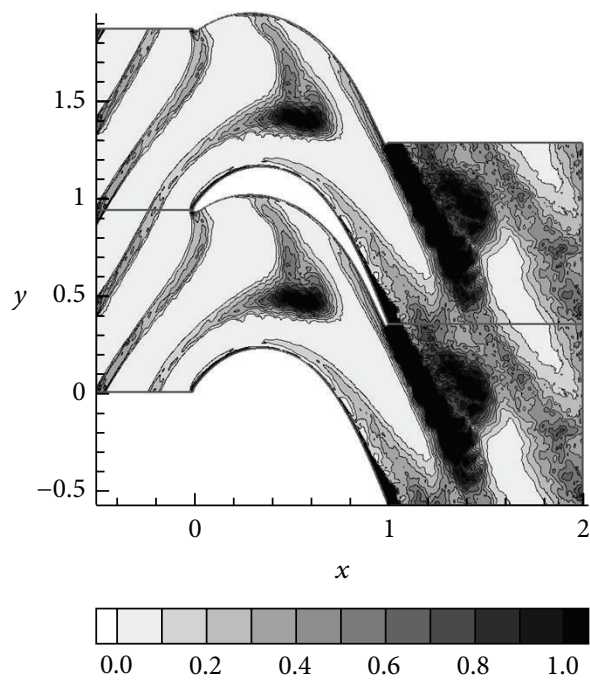

(c)
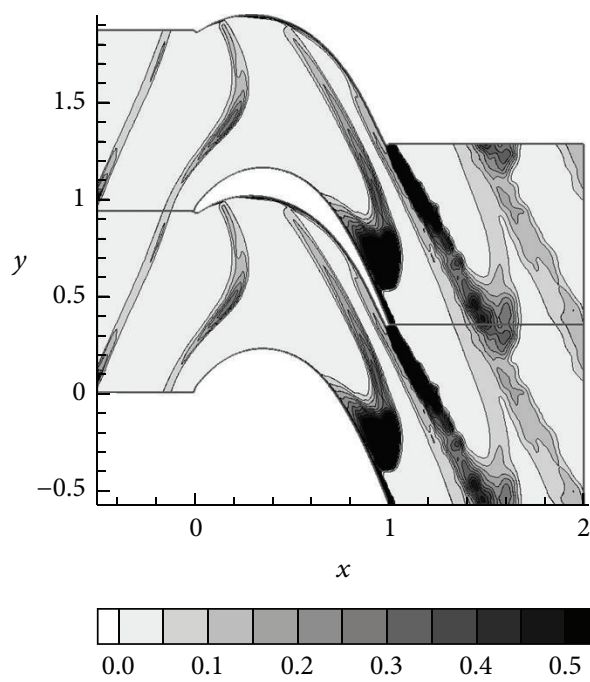

(e)

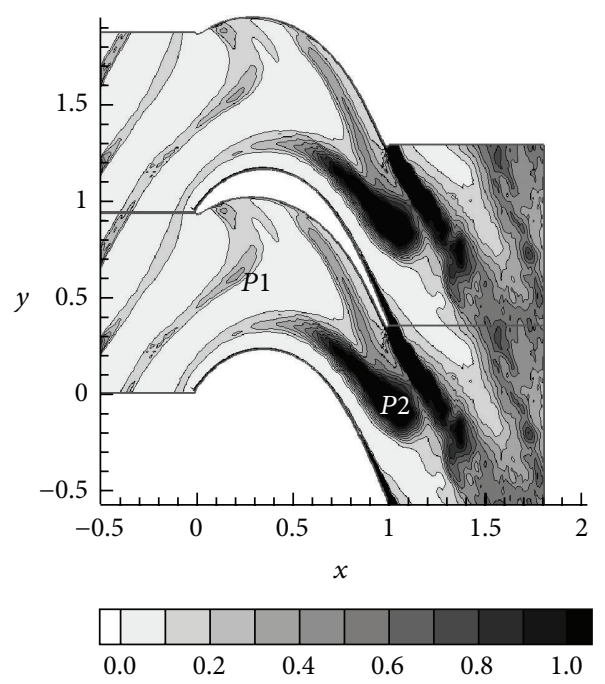

(b)

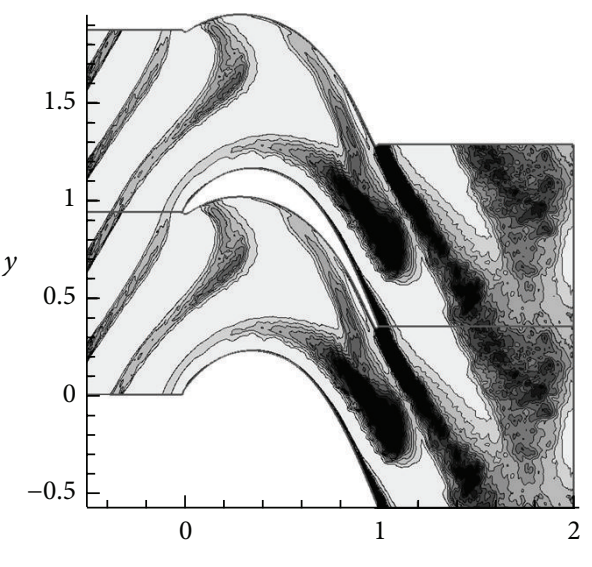

$x$

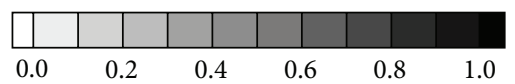

(d)

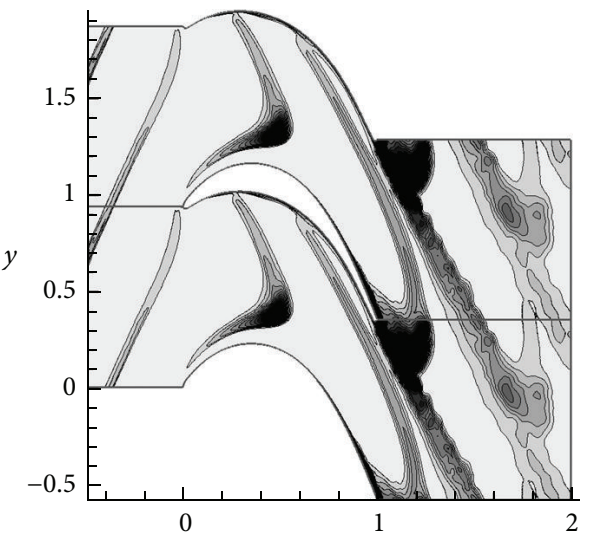

$x$

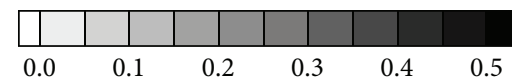

(f)

FIGURE 2: Phase-averaged turbulent kinetic energy: (a) LES: Re $=5.18 \times 10^{4}, \Phi=0.508$, (b) LES: Re $=5.18 \times 10^{4}, \Phi=0.008$, (c) DNS: Re = $5.18 \times 10^{4}, \Phi=0.508$, (d) DNS: Re $=5.18 \times 10^{4}, \Phi=0.008$, (e) LES: $\operatorname{Re}=1.48 \times 10^{5}, \Phi=0.375$, and (f) LES: $\operatorname{Re}=1.48 \times 10^{5}, \Phi=1.0$. 
kinetic energy increases while the wake is gradually turned and deformed inside the vane. The gradual increase of $k$ is approximately located around the wake apex.

Apparently, the turbulent kinetic energy keeps on growing while the wake travels from the leading to the trailing edge of the blade. This phenomenon does not seem to be very sensitive to the Reynolds number since the same trend is obtained at $\operatorname{Re}=5.18 \times 10^{4}$ as well as at $\operatorname{Re}=1.48 \times$ $10^{5}$. The only noticeable qualitative difference between the results obtained at different Reynolds numbers consists in the increased proximity of the peak of $k$ to the suction side of the blade at $\operatorname{Re}=1.48 \times 10^{5}$. This, however, is most likely a consequence of the different inflow angle that is employed in the simulations at $\mathrm{Re}=5.18 \times 10^{4}$ compared to those at $\operatorname{Re}=1.48 \times 10^{5}$. At the lower Reynolds number the inflow angle is larger and the core of the flow region with large values of $\langle k\rangle$ inside the vane never manages to hit the suction side boundary layer. Conversely, the wake region with increased $\langle k\rangle$ clearly hits the suction side boundary layer in the simulation with the larger Reynolds number and smaller inflow angle. The plots also indicate that the portion of the flow field with the large values of turbulent kinetic energy in the wake remains somewhat confined up to $x / C_{\mathrm{ax}} \cong 0.6-0.7$. Further downstream, while approaching the exit region of the flow, the spot with the large values of $k$ diffuses and merges with the wake downstream of the trailing edge. Here, another difference arises between the simulations for different inflow angles and Reynolds numbers: while for the higher-inflowangle simulation the incoming wake merges with the trailing edge wake towards the pressure side, for the smaller-inflowangle simulation the incoming and trailing edge wakes merge near the suction side.

The observed large values of turbulent kinetic energy appear to stem from large values of local production, as is illustrated in Figure 3 which shows the production of turbulent kinetic energy, computed by using the phaseaveraged quantities, $\left\langle P_{k}\right\rangle=-\left\langle\overline{u_{i} u_{j}}\right\rangle \cdot\left(\partial\left\langle U_{i}\right\rangle / \partial x_{j}\right)$. All plots reveal that the peak of turbulent kinetic energy coincides with large values of the production in the blade vane. While there is not much production in the portion of the wake between the pressure side and the apex, in the portion from the apex to the suction side $\left\langle P_{k}\right\rangle$ values are obtained up to 10 times as large as those encountered in the wake near the inlet section.

The circles on the lines " $P$ " of Figure 4 represent the approximate location of the phase-averaged turbulent kinetic energy peaks, observed in Figure 2, while the wake is swallowed into the blade vane for each of the three simulations. The path of line " $P$ " corresponds to the path of the bow apex of the wake, which also corresponds roughly to the location of the maximum wake curvature. Moreover, the analysis of the unsteady flow field reveals that the location of the peak follows the path of a fluid element in the wake, which moves towards the suction side on account of the cross-flow pressure gradient induced by the core flow turning. Figure 4 reveals that the peaks remain clearly outside the suction side boundary layer, although the turbulent kinetic energy clearly diffuses close to wall in proximity to the trailing edge. For $x / C_{\mathrm{ax}}>0.4-0.5$, the distance from the wall becomes virtually constant. This can also be observed in the contour plots of $k$ and $P_{k}$ in Figures 2 and 3.

Now that the peaks of turbulent kinetic energy in the vane are localised, it is desirable to show the magnitude of $k$ and $P_{k}$ along $P$ at different phases $\Phi$. This is done by interpolating the desired quantities along the fixed line $P$ at selected phases, and it is illustrated in Figure 5; the abscissa reports the axial coordinate $x$ to avoid problems stemming from the slightly different lengths of the $P$ curves for the three simulations. The plots start at $x / C_{\mathrm{ax}}=0$, which corresponds to the blade leading edge and stop shortly downstream of the trailing edge, located at $x / C_{\mathrm{ax}}=1$. For the $\mathrm{Re}=5.18 \times 10^{4}$ case, Figure 5 shows five discrete curves, which correspond to five phaseaveraged snapshots of the flow field equally spaced to cover one full period. They are selected from the database with 120 stored phases for the LES and 240 for the DNS.

For the $\operatorname{Re}=1.48 \times 10^{5}$ simulation the same figure reports four equally spaced phases out of the 64 stored phases. Both $k$ and $P_{k}$ are computed by using the phase-averaged flow fields. The dashed curves draw the envelope of the peaks at different phases of both the selected quantities along the $P$ line. The dashed curves show that the peak of turbulent kinetic energy increases while the wake is swallowed into the vane, and it begins to decrease slightly upstream of the trailing edge. Remarkably, this behaviour does not depend on the Reynolds number. For the $\mathrm{Re}=5.18 \times 10^{4}$ case, the peak of $k$ in the LES result is located somewhat upstream of the position found in the DNS. It must be pointed out that some of the differences in the curves between DNS and LES at $\operatorname{Re}=5.18 \times 10^{4}$ may stem from the difficulties in tracing the peak of $k$ and selecting exactly the same phase for the two simulations. Observe that each phase-averaged curve shows two peaks (see, e.g., the peaks indicated by the labels $P 1$ and $P 2$ in Figure 5(a)): the first one refers to the new incoming wake, whereas the second one is clearly the product of the previous wake which travels downstream. They correspond qualitatively to the wake peaks labelled $P 1$ and $P 2$ in Figure 2(b). The curves show that the peaks are quite sharp along the first 50 to $60 \%$ of the blade; that is, the large values of $k$ are localised in a narrow region. These peaks partly diffuse once the trailing edge of the blade is approached. An estimate of the growing strength of the localised peaks of $k$ along the wake path is given by the ratio of the turbulent kinetic energy encountered in the wake upstream of the blade, $k_{\text {wake }}$, and the maximum peak of $k$ encountered when the wake is inside the blade vane, $k_{\text {vane. }}$. For the time phases considered here the ratio $k_{\text {vane }} / k_{\text {wake }}$ can exceed the value of 10 . Conversely, the growth of the turbulence intensity (defined as the ratio of the square root of the local turbulent kinetic energy and the mean local velocity, $\mathrm{Tu}=\sqrt{(2 / 3) k} /|U|)$ is not as steep since $\mathrm{Tu}$ reaches values approximately 50 to $60 \%$ larger than the levels found deep inside the wake upstream of the cascade.

The production rates of turbulent kinetic energy, $P_{k}$, shown in Figure 5, also increase continuously while the wake is travelling in the first $50-60 \%$ of the blade vane. The plots reveal that the envelopes of the peaks of both $k$ and $P_{k}$ substantially coincide in the first 50 to $60 \%$ of the blade vane. This confirms the strong link between these two quantities, 

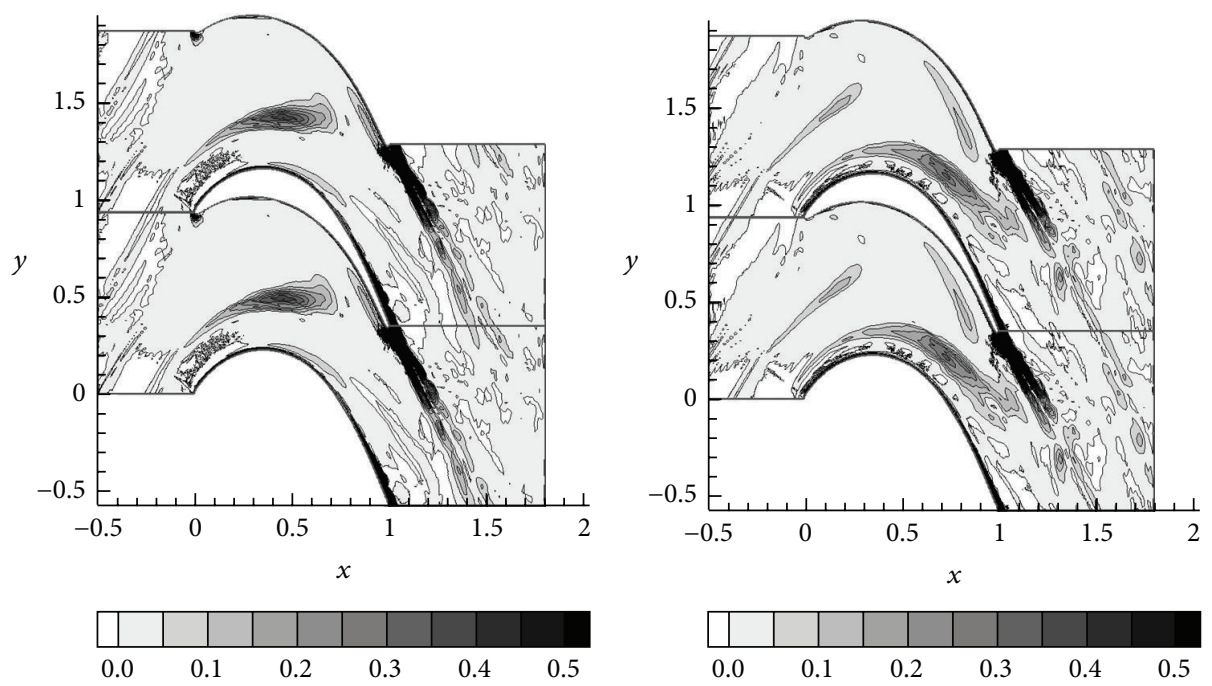

(a)

(b)
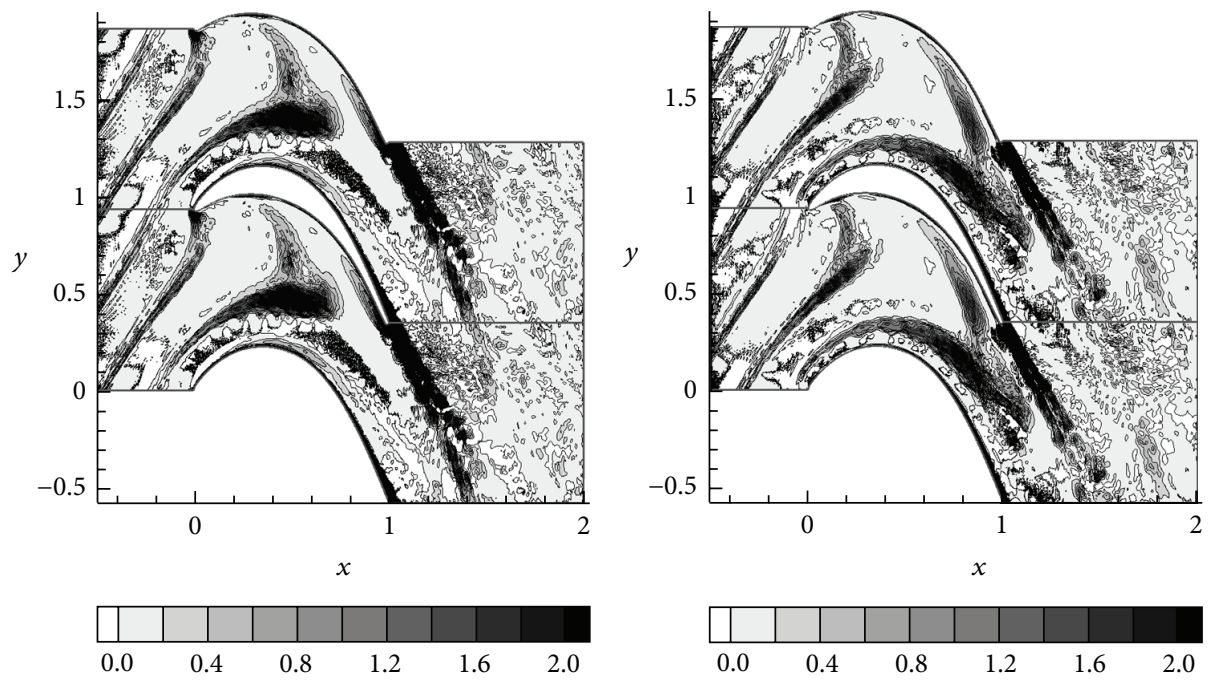

(c)

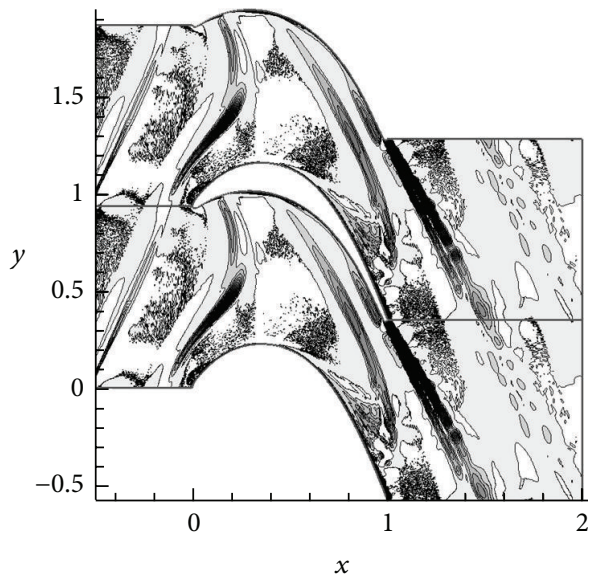

(d)
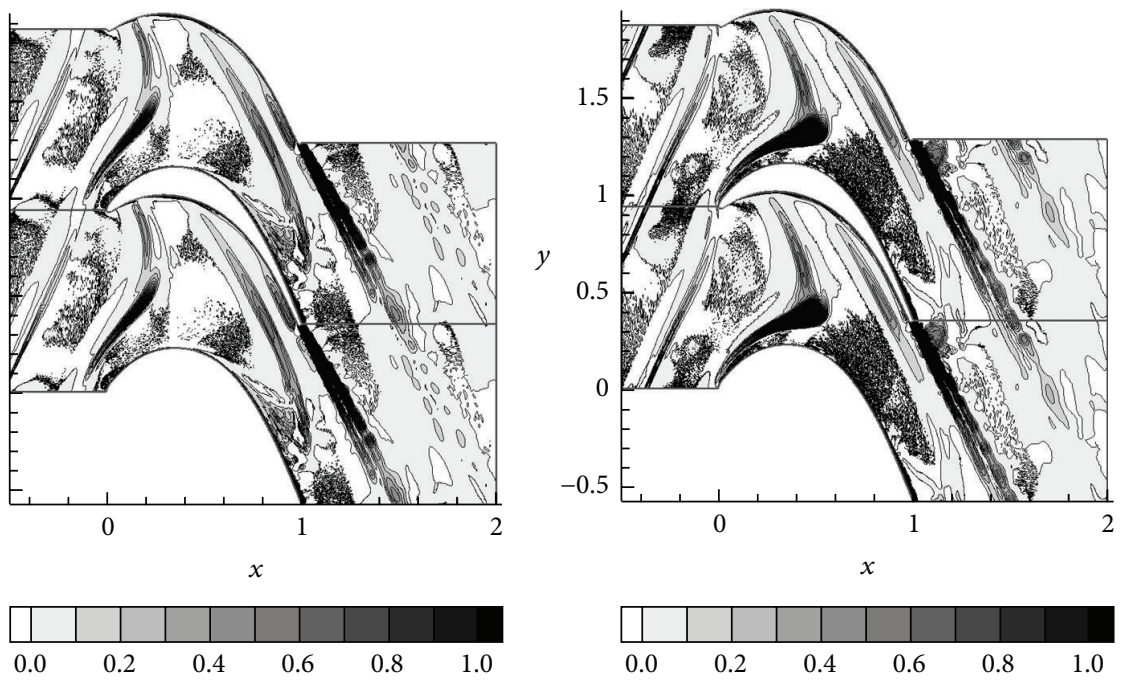

(e)

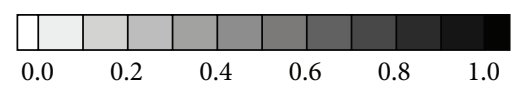

(f)

FIGURE 3: Phase-averaged production of turbulent kinetic energy: (a) LES: Re $=5.18 \times 10^{4}, \Phi=0.508$, (b) LES: Re $=5.18 \times 10^{4}, \Phi=0.008$, (c) DNS: $\operatorname{Re}=5.18 \times 10^{4}, \Phi=0.508$, (d) DNS: $\operatorname{Re}=5.18 \times 10^{4}, \Phi=0.008$, (e) LES: $\operatorname{Re}=1.48 \times 10^{5}, \Phi=0.375$, and (f) $\operatorname{LES}: \operatorname{Re}=1.48 \times 10^{5}$, $\Phi=1.0$. 


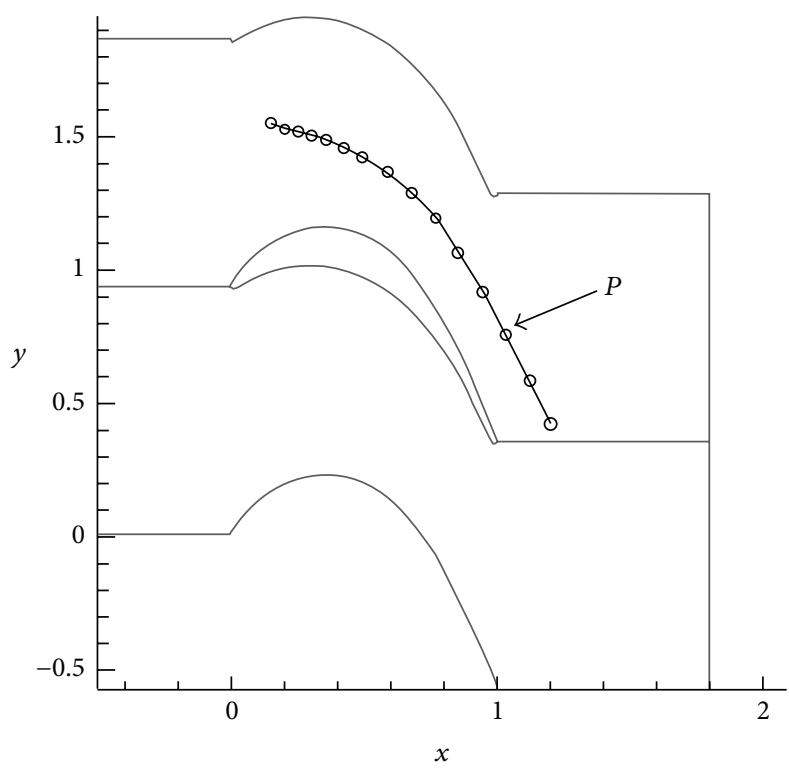

(a)

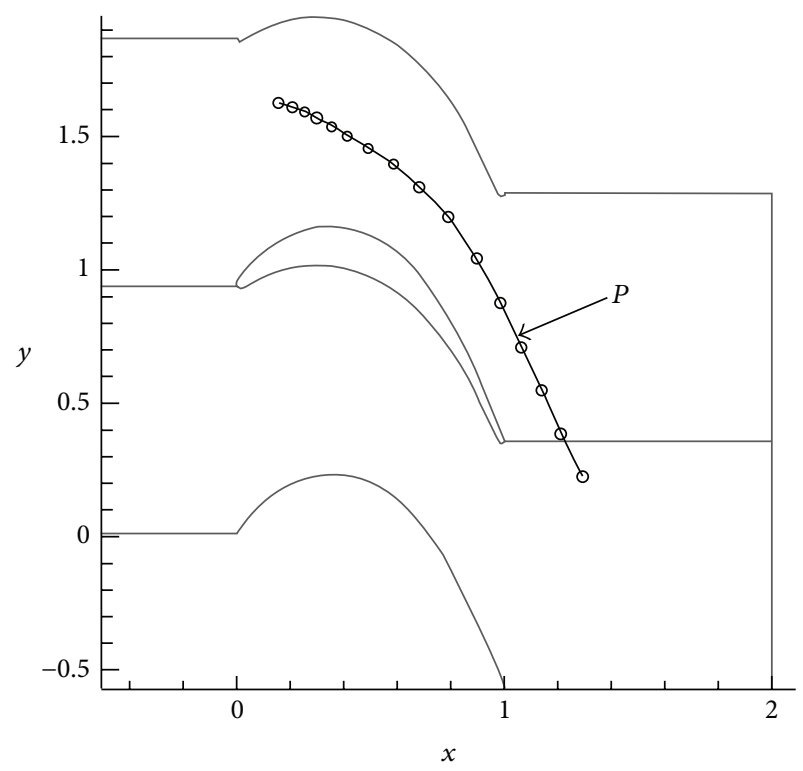

(b)

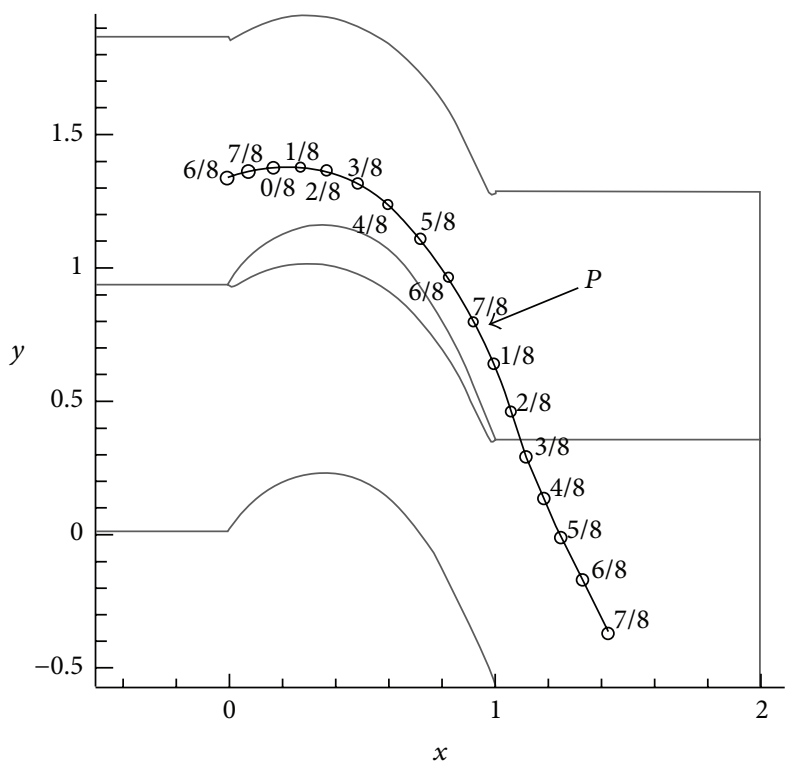

(c)

FIGURE 4: Approximate path of the peak of turbulent kinetic energy in the blade vane: (a) LES: Re $=5.18 \times 10^{4}$, (b) $\mathrm{DNS}$ : Re $=5.18 \times 10^{4}$, and (c) LES: $\operatorname{Re}=1.48 \times 10^{5}$.

regardless of the Reynolds number, of the wake frequency, and of the nature of the simulation (DNS or LES). Further downstream the rate of production of turbulent kinetic energy drops and, consequently, the dissipation of turbulent kinetic energy takes over. Hence, the gradient of $k$ reverts from positive to negative. The drop of $P_{k}$ is the strongest in the $\operatorname{Re}=1.48 \times 10^{5}$ case.

Now that the peaks of $k$ and $P_{k}$ have been clearly identified in both space and time by using the phase-averaged flow fields, it is possible to show how they are linked to the strain and stress tensors. Prior to doing this it is convenient to recall the expression for the phase-averaged production rate:

$$
\left\langle P_{k}\right\rangle=-\left\langle\tau_{i, j}\right\rangle \cdot\left\langle S_{i, j}\right\rangle
$$

in which $\left\langle\tau_{i, j}\right\rangle$ and $\left\langle S_{i, j}\right\rangle$ are the turbulent stress and strain tensors, respectively. The relative contribution of strain and stress to the production rate can be quantified by computing the eigenvalues of the two tensors. Analysing the 2D strain 

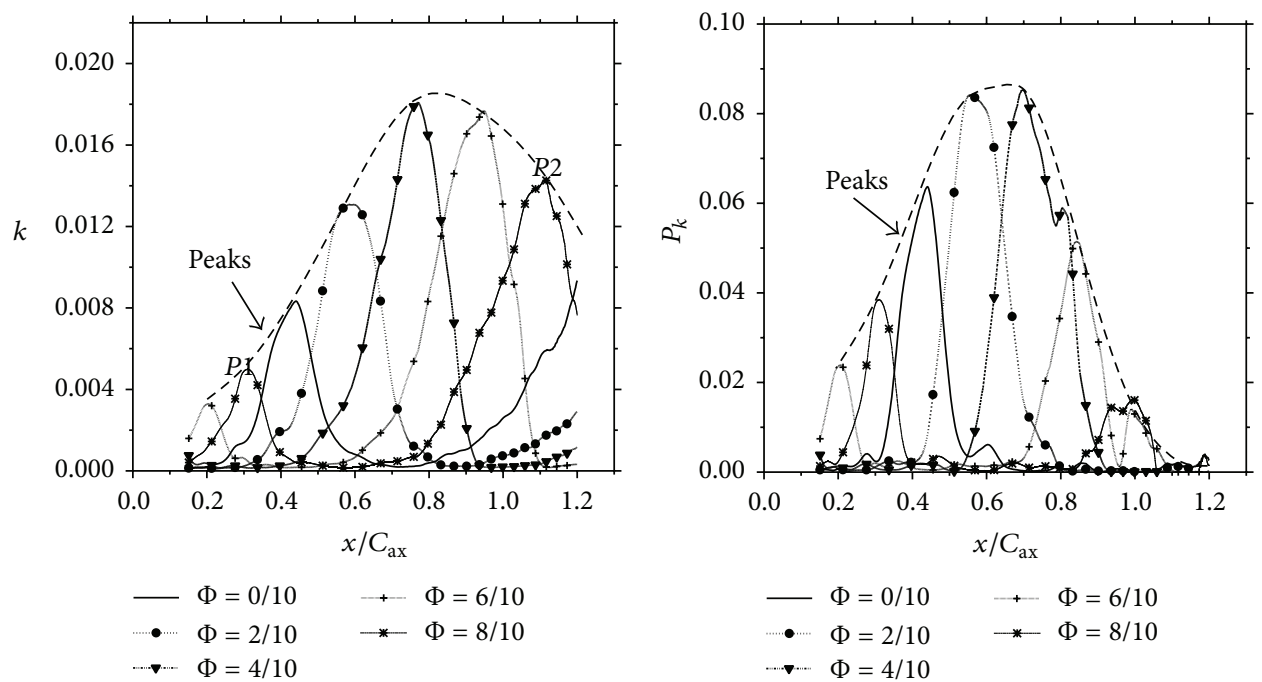

(a)
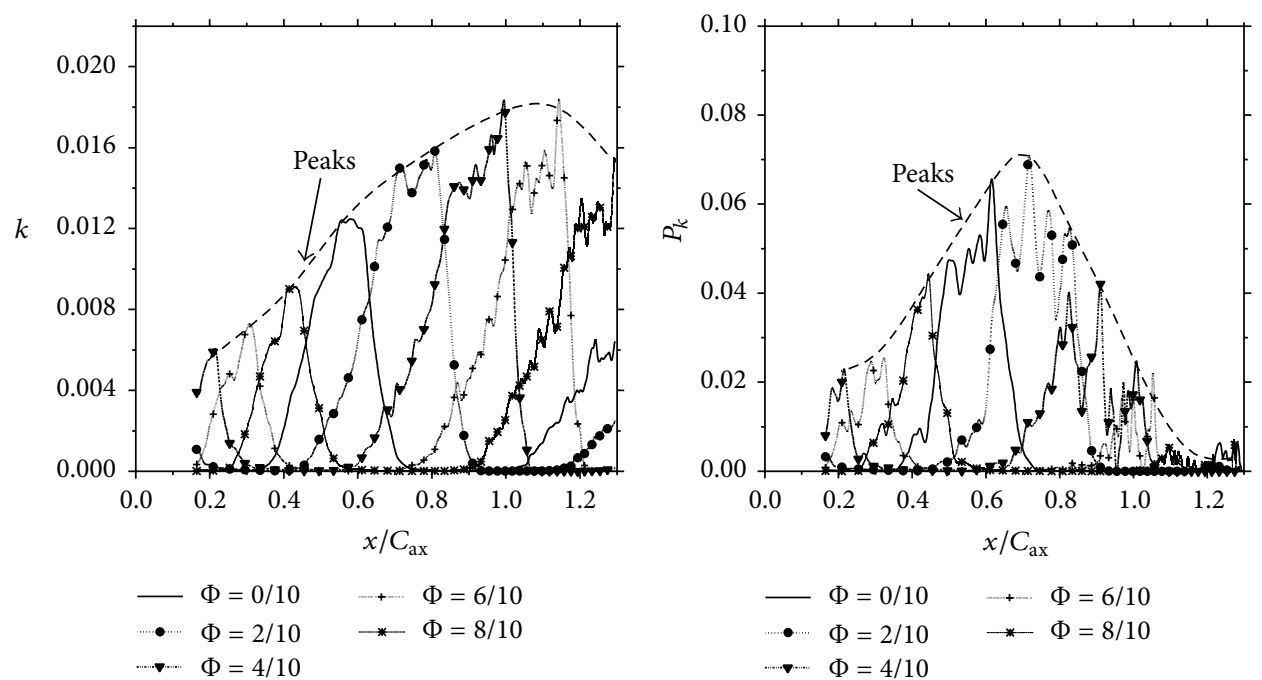

(b)
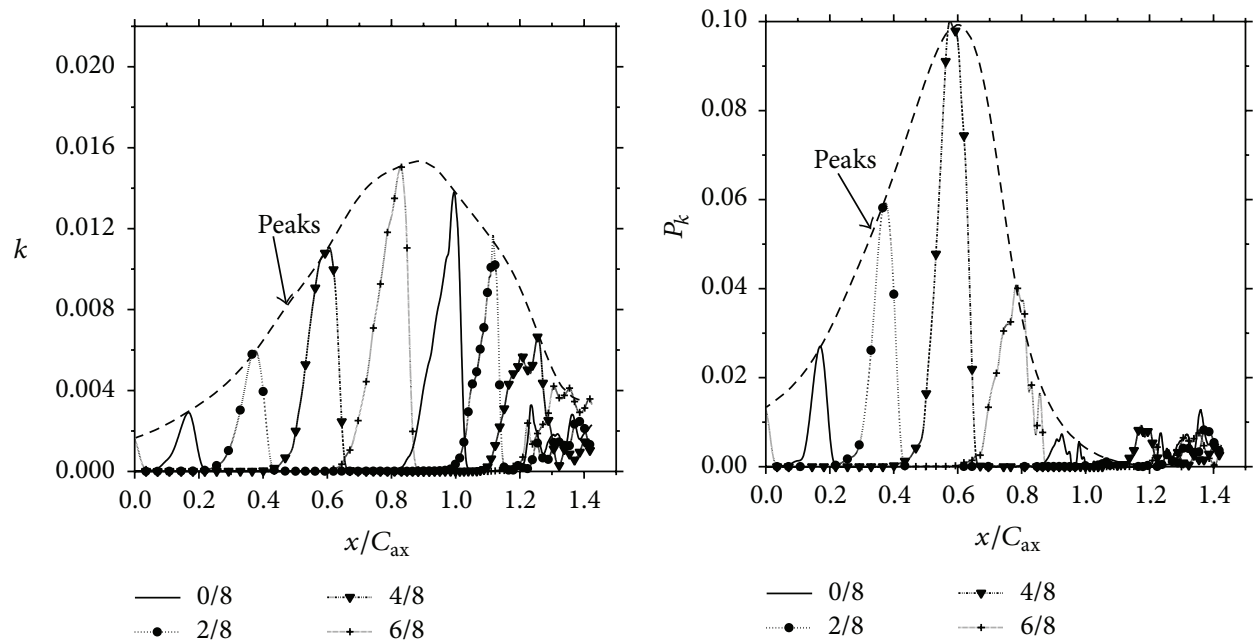

(c)

Figure 5: Peaks of turbulent kinetic energy, $k$, and production rate, $P_{k}$, along line $P$ versus the axial coordinate in the blade vane: (a) LES: Re $=5.18 \times 10^{4}$, (b) DNS: $\operatorname{Re}=5.18 \times 10^{4}$, and (c) LES: $\operatorname{Re}=1.48 \times 10^{5}$. 


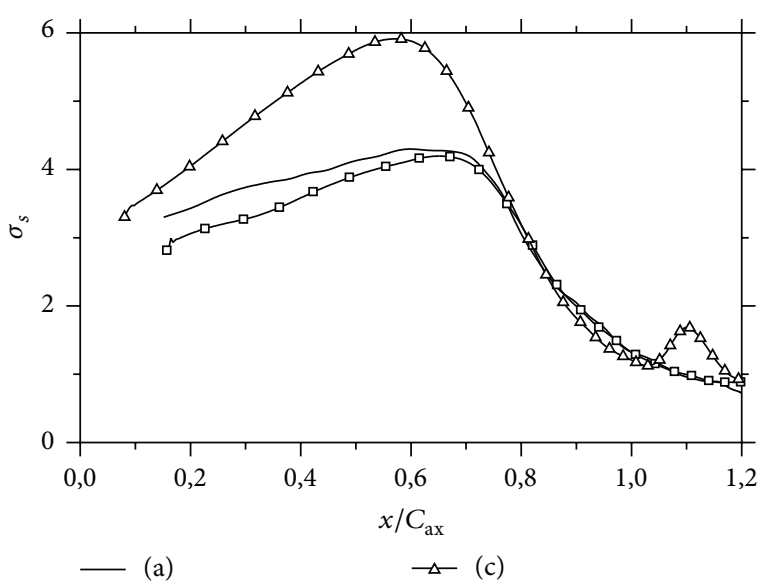

$\rightarrow-(b)$

FIGURE 6: Time averaged magnitude of the strain tensor eigenvalue along line $P$ versus the axial coordinate in the blade vane: (a) LES: $\mathrm{Re}=5.18 \times 10^{4}$, (b) DNS: Re $=5.18 \times 10^{4}$, and (c) LES: Re $=1.48 \times$ $10^{5}$.

tensor instead of the 3D tensor suffices since we assume spanwise flow periodicity and because of the prismatic nature of the blade (i.e., in the phase-averaged flow field $S_{3,3}=0$ ). Since the flow is incompressible, implying that $\operatorname{tr}\left(S_{i, j}\right)=0$, the $2 \mathrm{D}$ strain tensor can be rewritten along its principal axes as

$$
\Lambda_{S}^{2 \mathrm{D}}=\left(\begin{array}{cc}
\sigma_{s} & 0 \\
0 & -\sigma_{s}
\end{array}\right)
$$

The same analysis can be carried out for the stress tensor. In this case the $3 \mathrm{D}$ tensor for the current flow configurations reads

$$
\left\langle\tau_{i, j}\right\rangle=\left(\begin{array}{ccc}
\left\langle u_{1} u_{1}\right\rangle & \left\langle u_{1} u_{2}\right\rangle & 0 \\
\left\langle u_{2} u_{1}\right\rangle & \left\langle u_{2} u_{2}\right\rangle & 0 \\
0 & 0 & \left\langle u_{3} u_{3}\right\rangle
\end{array}\right)
$$

in which $\left\langle u_{3} u_{1}\right\rangle=\left\langle u_{1} u_{3}\right\rangle=\left\langle u_{3} u_{2}\right\rangle=\left\langle u_{2} u_{3}\right\rangle=0$ because of span-wise periodicity. Therefore, for the present analysis once again it suffices to refer to the $2 \mathrm{D}$ subtensor since there is no direct phase-averaged production of turbulence from span-wise stresses. The third eigenvalue is $\left\langle u_{3} u_{3}\right\rangle$, which corresponds to the third eigenvector aligned with the spanwise direction. Hence, the 2D substress tensor reads

$$
\left\langle\tau_{i, j}^{2 \mathrm{D}}\right\rangle=\left(\begin{array}{ll}
\left\langle u_{1} u_{1}\right\rangle & \left\langle u_{1} u_{2}\right\rangle \\
\left\langle u_{2} u_{1}\right\rangle & \left\langle u_{2} u_{2}\right\rangle
\end{array}\right)
$$

The two-dimensional stress tensor eigenvalues read

$$
\Lambda_{\tau}^{2 \mathrm{D}}=\left(\begin{array}{cc}
\sigma_{\tau}^{1} & 0 \\
0 & \sigma_{\tau}^{2}
\end{array}\right)
$$

Now, the magnitude of the eigenvalues along the $P$ line allows investigating what is the contribution of the strain and turbulent stress tensors to the production rate of (1) in the plane $x-y$. Most likely due to the limited number of phases used for the phase-averaging, the eigenvalues, $\sigma_{S}$ and $-\sigma_{S}$,

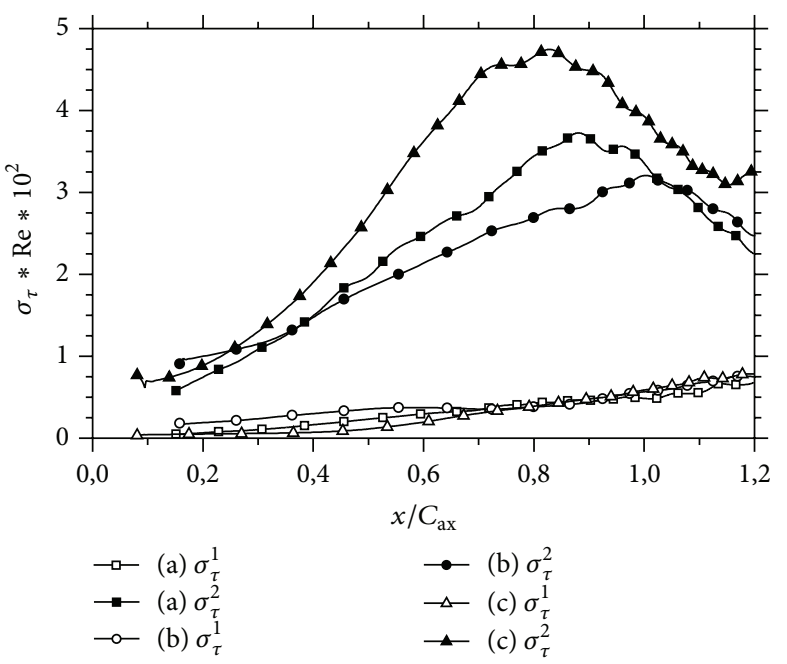

FIGURE 7: Time averaged magnitude of the stress tensor eigenvalue along line $P$ versus the axial coordinate in the blade vane: (a) LES: $\operatorname{Re}=5.18 \times 10^{4}$, (b) DNS: $\operatorname{Re}=5.18 \times 10^{4}$, and (c) LES: $\operatorname{Re}=1.48 \times$ $10^{5}$.

and $\sigma_{\tau}^{1}$ and $\sigma_{\tau}^{2}$ of the phase-averaged $2 \mathrm{D}$ strain tensor and stress tensor, respectively, extracted along the $P$ line show a somewhat chaotic behaviour. Hence, it was decided to plot the average magnitude of the phase-averaged eigenvalues along the $P$ line for the three simulations, as shown in Figures 6 and 7. The curves reveal that the peak of the production rate roughly corresponds to the maximum of the largest strain tensor eigenvalue, while the peak of the stress tensor eigenvalue, although quite close, is located $0.10-0.15 \times$ $C_{\mathrm{ax}}$ downstream. Moreover, the overall shape of the largest strain tensor eigenvalue curve resembles the shape of the turbulence production curve plotted in Figure 5. Apparently, the turbulent stress reacts to the turning and straining of the wake with some slight delay with respect to the strain tensor. This feature was already observed by Rogers [5] for linear wakes in which either the direction of compression or that of stretching was aligned with the wake path.

\section{Analysis along the Wakes}

The previous section proved that the peak of production is located close to the strain tensor eigenvalue peak and substantially stems from the concerted action of the peaks of both strain and turbulent stresses. In order to understand how the wake triggers both production and turbulent kinetic energy, it is convenient to concentrate the analysis on a line aligned with the phase-averaged wakes. For this purpose we selected two different phases, $\Phi=0.008$ and 0.508 for the $\operatorname{Re}$ $=5.18 \times 10^{4}$ case and $\Phi=0.375$ and 1.00 for the $\mathrm{Re}=1.48 \times$ $10^{5}$ case, respectively. The lines that identify the wakes follow the location of the maximum velocity magnitude defect and are illustrated in Figure 8. The 2D phase-averaged strain tensor eigenvectors allow to identify the local directions of compression and stretching and to compare these directions with those of the eigenvectors of the stress tensor along the 

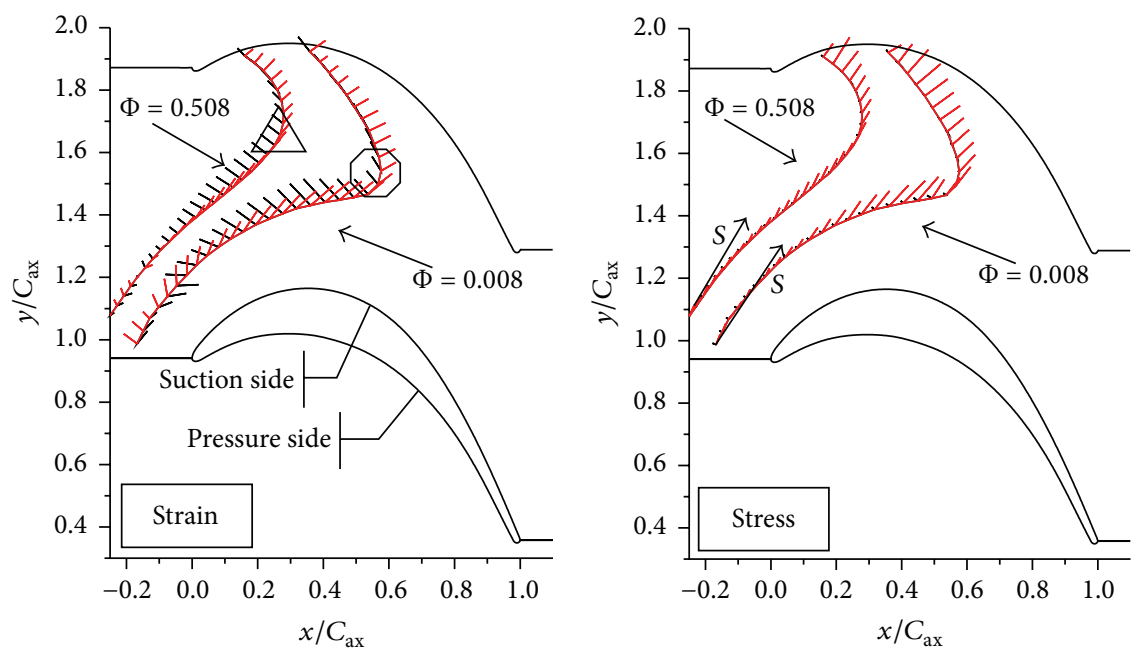

(a)
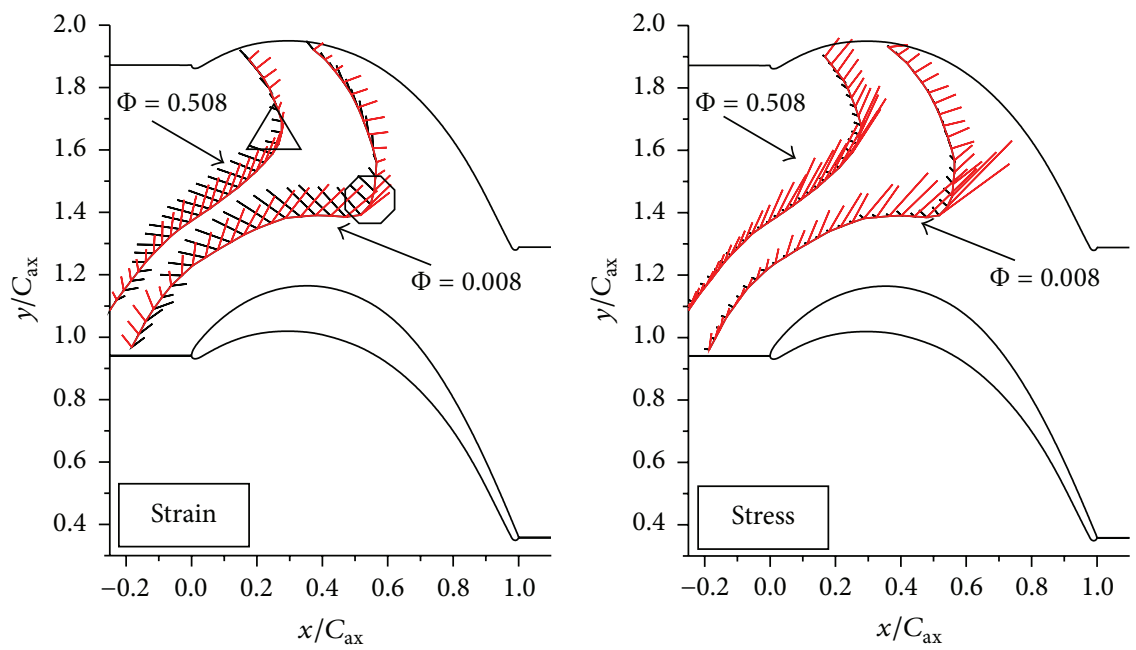

(b)
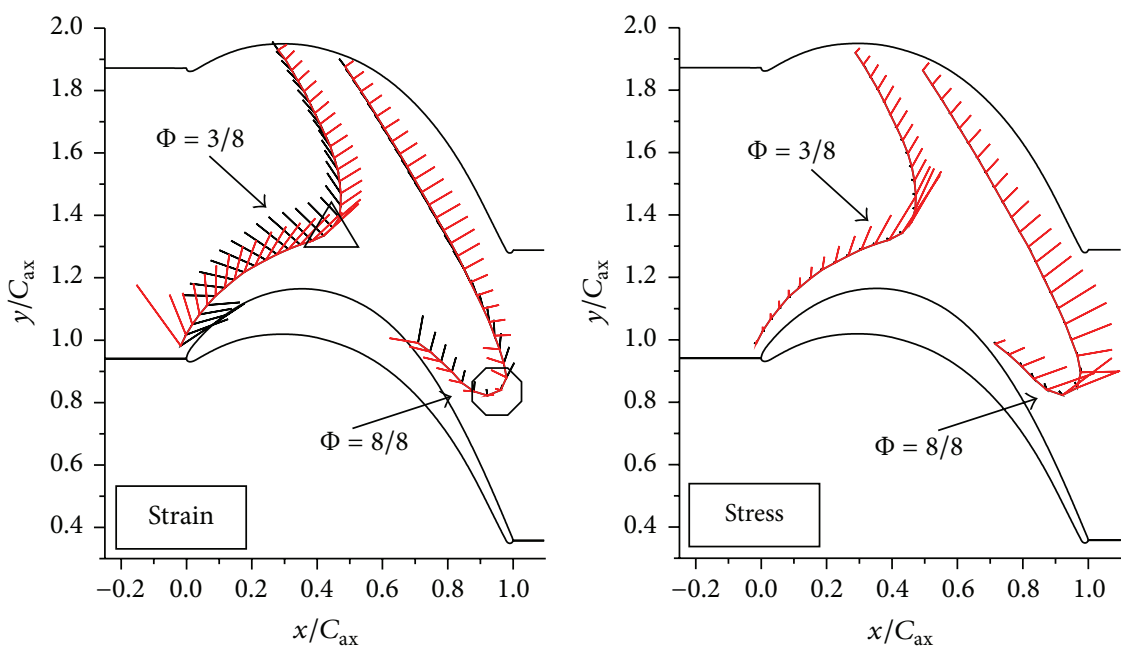

(c)

FIGURE 8: Eigenvectors of the strain tensor (left) and of the stress tensor (right) along two selected wakes: (a) LES: Re $=5.18 \times 10^{4}$, (b) DNS: $\operatorname{Re}=5.18 \times 10^{4}$, and (c) LES: $\operatorname{Re}=1.48 \times 10^{5}$ (for the strain tensor, the red segment represents the direction of compression). 


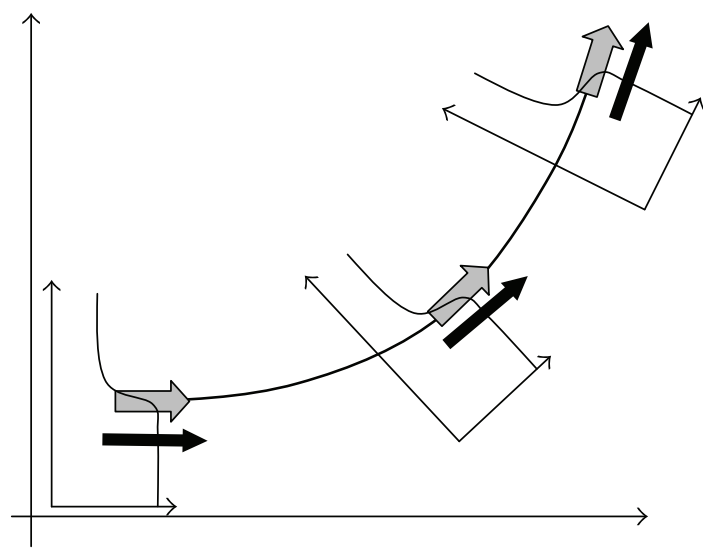

(a) Castro and Bradshaw [2]

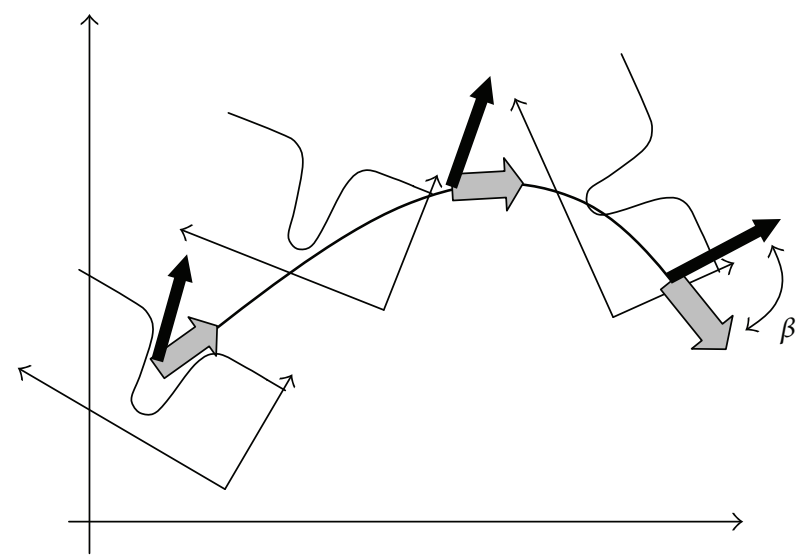

(b) Present

FIGURE 9: Sketch of the convection and shear layer directions. Grey arrows indicate direction of convection; thick black arrows indicate orientation of shear layer in proximity to the bow apex of the incoming wakes.

selected wakes. This comparison is carried out in Figure 8 for both the LES and the DNS at Re $=5.18 \times 10^{4}$ and the LES at Re $=1.48 \times 10^{5}$. Observe that the eigenvector length is chosen to be proportional to the corresponding eigenvalue magnitude. The plots show a remarkable feature of flow; the wake is almost perfectly aligned with the direction of compression in the proximity of its apex. This seems to be a common feature for both wake positions and for both values of the Reynolds numbers, regardless of the nature of the simulation (DNS or LES). Wu and Durbin [6] observed the same feature in their DNS of the same flow at $\operatorname{Re}=1.48 \times 10^{5}$.

Apparently, while the wake is smoothly turned and deformed inside the blade vane, the portion which is entrained in the fastest part of the flow field, identified by the bow apex of the wake, gradually aligns with the direction of compression. The portion of the wake, shown in Figure 8, close to the suction side is not aligned with either the direction of compression or that of stretching, whereas the portion from the apex to the pressure side is almost aligned with the direction of stretching.

The plots showing the eigenvectors of the stress tensor along the wakes reveal a similar behaviour; one of the eigenvectors of the stress tensor is clearly tangent to the wake in proximity to its apex. This is again a common feature of the two Reynolds numbers and the two wake positions, and, at least for the lower Reynolds number case, it is predicted by both DNS and LES. Observe that, of the two, the eigenvector that is almost aligned with the wake is the one associated with the largest eigenvalue. This interesting feature of the flow was also observed for all other phase-averaged positions of the wake, and it is not reported here for the sake of brevity. Figures 4 and 7 reveal that the location of the turbulent kinetic energy peaks corresponds to the apex of the wakes. On turn the apex of the wakes is almost perfectly aligned with one of the eigenvectors of both the strain and stress tensors. In other words the location of the turbulent kinetic energy peak corresponds to the wake-eigenvectors alignment.
The production rate of turbulent kinetic energy can be split into normal and tangential contributions, respectively. Unfortunately, in such a complex flow field the definition of normal and tangential production is not straightforward because of the relative motion of the wakes with respect to the background flow. Castro and Bradshaw [2] analysed a highly curved mixing layer and Gibson and Rodi [3] and Leschziner and Rodi [16] formulated the rate of production of turbulent kinetic energy in terms of normal and tangential stresses contributions (i.e., production that stems from normal and tangential stresses, resp.) for such flow. In the experiments by Castro and Bradshaw and in the annular and twin parallel jets computed by Leschziner and Rodi, the direction of convection coincides with the direction of the shear layer, as illustrated in Figure 9. In this case the decomposition between normal and tangential production is trivial and can be carried out by following Gibson and Rodi. However, in the present flow configuration the direction of convection differs from the direction of the shear layer generated by the incoming wake (Figure 9). In particular, the angle between the two directions at the inlet section can be as large as 30 degrees, and it reaches approximately 90 degrees inside the blade vane. This is made evident in Figure 10, which shows on the same plot at $\Phi=0.00$ both the streamlines and the velocity defect defined as $u_{d}=\sqrt{\left(\overline{u_{1}}-\left\langle u_{1}\right\rangle_{0}\right)^{2}+\left(\overline{v_{2}}-\left\langle v_{2}\right\rangle_{0}\right)^{2}}$.

In order to determine whether the large production rate in proximity to the wake apex is mostly due to normal or tangential stresses, the particular orientation of both the strain and stress tensors eigenvectors with respect to the wake needs to be further investigated. Prior to this it is convenient to analyse what happens in a turbulent boundary layer as a simplified reference condition. This is done in Figure 11, where the typical eigenvectors of a turbulent boundary layer refer to the log-law region with moderate anisotropy. Here, the shear layer is aligned with the wall and with the direction of the core flow. Due to incompressibility, the strain tensor eigenvectors in the turbulent boundary layer are such that 


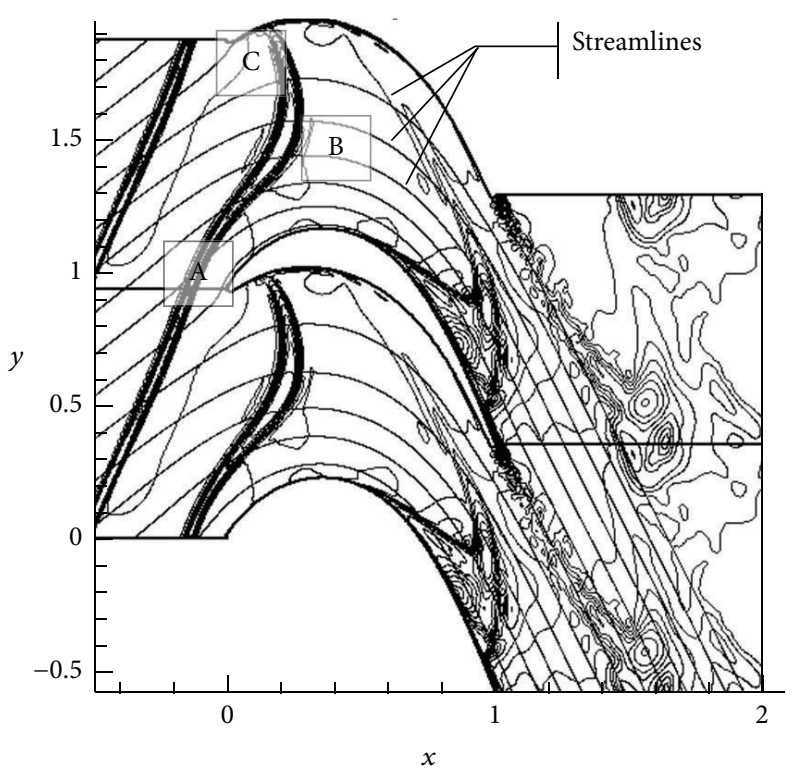

FIGURE 10: Phase-averaged streamlines and velocity defect isolines computed by the LES, $\Phi=0.00, \operatorname{Re}=1.48 \times 10^{5}$.

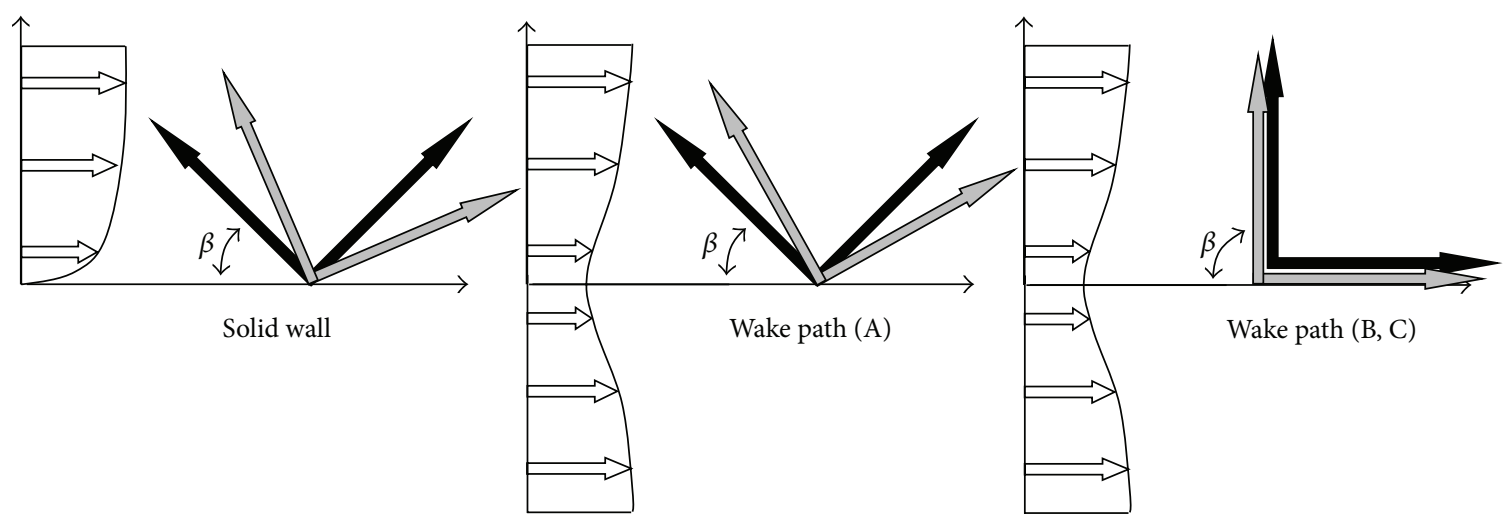

FIGURE 11: Sketch of the strain tensor eigenvectors (black) and stress tensor eigenvectors (grey) in a turbulent boundary layer (left), and in two positions $\mathrm{A}$ and $\mathrm{B}$, as detailed in Figure 10, along a typical wake.

$\beta=45$ deg while for the stress tensor eigenvectors $\beta<$ $45 \mathrm{deg}$ and the production rate are due to tangential stresses (see Pope [17]). The strain and stress tensor eigenvectors for the wake are extracted from the points labelled A and B as documented in Figure 10. The wake in the turbine vane can be imagined as the sum of two virtually parallel shearlayers, as illustrated in Figures 11(A) and 11(B, C). The rotation of the core flow only produces a very weak shear whose effect on the local production of turbulence can be neglected when compared to the effect of the wake shear layers. In point A (see Figure 11(A)), which is located away from the apex and close to the suction side the orientation of the eigenvectors with respect to the wake is very similar to what is found for the boundary layer: both the strain and stress tensors eigenvectors are approximately at an angle $\beta$ of $40-$ 45 deg with the wake and, consequently, with the main shear layers. Hence, the production rate is mostly governed by shear stresses. In point $\mathrm{B}$ the situation drastically changes: one of the eigenvectors of both the strain and stress tensors is almost aligned with the shear layer produced by the wake (see Figure 11(B)). This means that both tensors are diagonal in a reference frame aligned with the wake shear layer. Therefore, the absence of any significant contribution stemming from off-diagonal terms suggests that in point $B$ the production of turbulence is predominantly due to normal strain. In particular, it is the eigenvector that identifies the direction of compression that aligns with the bow apex of the wake. In point $C$ the eigenvectors are still almost aligned with the wake, but here it is the direction of stretching that aligns with the shear layer. Hence, the strong straining of the shear layer considerably reduces the production rate with respect to point $\mathrm{B}$. This is particularly evident for the first of the two wake positions reported in Figure $12(\Phi=0.008$ at $\operatorname{Re}=5.18$ $\times 10^{4}$ and $\Phi=0.375$ at $\left.\operatorname{Re}=1.48 \times 10^{5}\right)$. In fact, after the peak located at $S / S_{\max } \cong 0.5, P_{k}$ drops down while approaching the pressure side.

It is now possible to rearrange the production rate in the principal frame of reference identified by the eigenvectors 

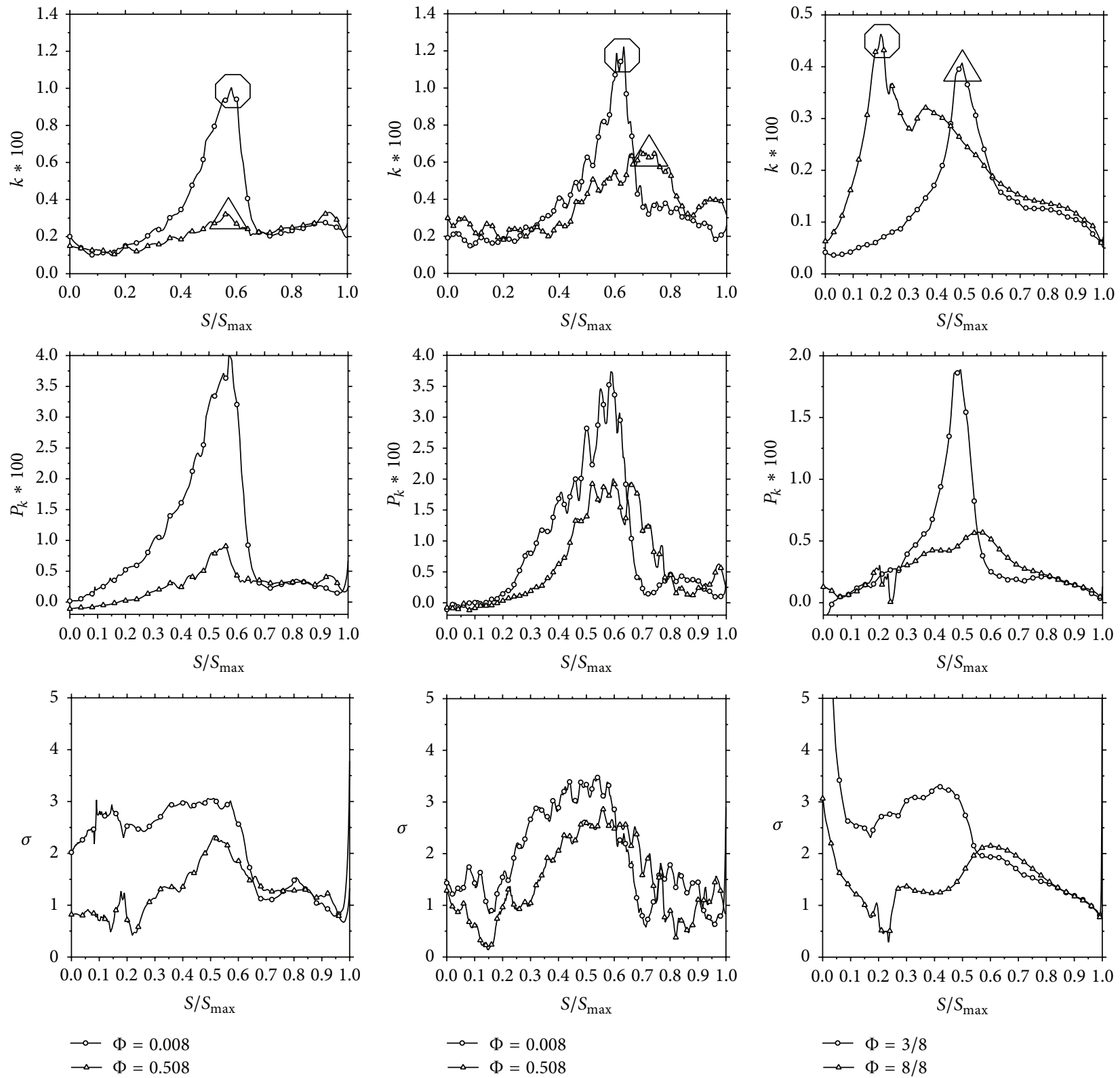

(a)

(b)

(c)

FIGURE 12: Turbulent kinetic energy, $k$, production rate, $P_{k}$, and strain tensor eigenvalue, $\sigma$, along two selected wakes: $(\mathrm{a}) \mathrm{LES}$ : $\operatorname{Re}=5.18 \times 10^{4}$, (b) DNS: $\operatorname{Re}=5.18 \times 10^{4}$, and (c) LES: $\operatorname{Re}=1.48 \times 10^{5}$.

of the strain tensor, and not in the direction of the flow. By defining $\alpha$ as the angle between the $x$-axis and the direction of compression, the stress tensor components, $\tau_{i j}$, are

$$
\begin{gathered}
\left\langle u_{p}^{2}\right\rangle=\left\langle u^{2}\right\rangle \cdot \cos ^{2} \alpha+\left\langle v^{2}\right\rangle \cdot \sin ^{2} \alpha+2 \cdot\langle u v\rangle \cdot \sin \alpha \cdot \cos \alpha, \\
\left\langle v_{p}^{2}\right\rangle=\left\langle u^{2}\right\rangle \cdot \sin ^{2} \alpha+\left\langle v^{2}\right\rangle \cdot \cos ^{2} \alpha-2 \cdot\langle u v\rangle \cdot \sin \alpha \cdot \cos \alpha, \\
\left\langle u_{p} v_{p}\right\rangle=\left\langle v_{p} u_{p}\right\rangle=\left(-\left\langle u^{2}\right\rangle+\left\langle v^{2}\right\rangle\right) \cdot \sin \alpha \cdot \cos \alpha \\
+\langle u v\rangle \cdot\left(\cos ^{2} \alpha-\sin ^{2} \alpha\right)
\end{gathered}
$$

in which the subscript " $p$ " denotes values in the principal frame of reference. In the same frame, the strain tensor is obviously diagonal so that the production rate can be computed by a term by term multiplication of (2) and (6). The following normal stress contribution results in

$$
P_{k}^{N}=P_{k}=-\left[\sigma_{S} \cdot\left\langle u_{p}^{2}\right\rangle-\sigma_{S} \cdot\left\langle v_{p}^{2}\right\rangle\right] .
$$

As it could be expected, the contribution of the tangential stresses to the production rate in the principal frame of reference is analytically zero since

$$
P_{k}^{T}=-\left[\sigma_{S} \cdot\left\langle u_{p} v_{p}\right\rangle-\sigma_{S} \cdot\left\langle u_{p} v_{p}\right\rangle\right]=0 .
$$


Therefore, the production rate is mostly due to normal stresses where the wake is aligned with one of the principal directions.

It is now possible to plot $k, P_{k}$, and $\sigma_{S}$ along a phaseaveraged wake-wise coordinate $S$, defined in Figure 8(a), for both phase-averaged positions of the wakes illustrated in the same figure. The origin of $S$ is located in the proximity of the suction side. Figure 12 shows the values of $k$ along the two wakes. The circles and triangles help in locating the peak of $k$ along the wakes of Figure 8. It is evident that the peak of $k$ and the largest production of turbulent kinetic energy are approximately positioned at the point where the wake is aligned with the direction of compression and with one of the eigenvectors of the stress tensor. Both $k$ and $P_{k}$ gradually increase along the axis of the wake, starting from the suction side portion. They both reach a maximum and then decrease in the flow region where the wake is almost perfectly aligned with the direction of stretching.

For the $\operatorname{Re}=5.18 \times 10^{4}$ case, along the first wake $(\Phi=$ 0.508), compared to DNS, LES underpredicts $k$ and $P_{k}$. The reason for this may be partly the omission of SGS contributions in the LES. As remarked before, the maximum value of $k$ is generally located closer to the suction side boundary layer than in the lower Re case. When switching to the second wake $(\Phi=0.008)$, the discrepancies between DNS and LES are substantially reduced. A possible explanation is that at the previous phase there are not many significant flow structures and inside the wake mostly small scale fluctuations are present, which are not resolved, but modelled, by LES (and neglected in the plots). When moving further in time, turbulence has developed and more flow structures appear (as evidenced by the flow visualisation), the contribution of which is directly captured by the LES. The plots related to the flow at $\mathrm{Re}=1.48 \times 10^{5}$ also reveal another interesting point. At $\Phi=0.375$ the location of the peaks of $k$ and $P_{k}$ coincides, but at $\Phi=1.0$ the location of the large value of $k$ is different from that of $P_{k}$. This apparently contradicts what has been discovered so far. However, Figure 12(c) reveals that the maximum production rate at $\Phi=1.0$ is approximately $1 / 4$ of that at $\Phi=0.375$. In other words, the build-up of turbulence is virtually complete when the apex of the wake reaches $x / C_{\mathrm{ax}} \cong 0.9$ at $\Phi=1.0$ (Figure 8 ). Therefore, the peak of turbulent kinetic energy visible along this wake at $s / S_{\max } \cong 0.2$ (i.e., very close to the suction side) is the result of convection from upstream where the flow experiences large production rates.

\section{Conclusions}

The analysis conducted on both the DNS and LES data sets at two Reynolds numbers illustrates how the alignment of the wake with the strain tensor eigenvectors controls the position of the peak of turbulent kinetic energy production, while its strength is mainly controlled by the magnitude of the strain tensor eigenvalue. In practice the largest production of turbulent kinetic energy will take place at a particular position along the wake where the maximum strain tensor eigenvalue approximately coincides with the location of the alignment of the wake with the direction of compression. If this condition is not fulfilled, the turbulent kinetic energy may still grow locally (in fact the peak of turbulent kinetic energy is constantly increasing up to $\left.x / C_{\mathrm{ax}}=0.85-0.9\right)$ but at a smaller rate.

LES was also found in fair agreement with the DNS at $\operatorname{Re}=5.18 \times 10^{4}$, suggesting that further analyses may be conducted by using much less computationally intensive LES as compared to DNS, provided the discretization quality is carefully controlled to appropriately resolve not only the boundary layers, but the incoming wakes as well.

The high-fidelity simulation data sets identified all the mechanisms contributing the production rate of turbulent kinetic energy. The analysis showed the relative importance of the strain and stress tensors, respectively, together with normal and tangential contribution to the production rate by comparing with Castro and Bradshaw results. A further analysis carried out in the strain tensor principal frame of reference showed the main contributions to the production rate of turbulent kinetic energy.

The simulations also revealed differences in the location and intensity of the turbulent kinetic energy peaks when increasing the Reynolds number from $5.18 \times 10^{4}$ to $1.48 \times$ $10^{5}$ and incidence angle. In particular, at $\mathrm{Re}=1.48 \times 10^{5}$ the turbulent kinetic energy peak moves much closer to the suction side boundary layer. This phenomenon, associated with the local growth of turbulent kinetic energy above the free-stream level, is expected to improve the suction side boundary layer stability and it can provide very valuable information in the design of suction-side-separation-free low-pressure turbine blades.

Last, but not least, the anisotropic turbulence production rate captured by the DNS and LES can be compared with the prediction of lower order models, to allow fixing eventual weaknesses by a strictly analytical manner.

\section{Conflict of Interests}

The authors declare that there is no conflict of interests regarding the publication of this paper.

\section{Acknowledgments}

The authors would like to thank Professor W. Rodi for the fruitful discussions on the analysis of the results. This work was supported by the German Research Foundation (DFG) within the joint Project "Periodic Unsteady Flow in Turbomachinery."

\section{References}

[1] H. Schlichting, Boundary Layer Theory, McGraw-Hill, 7th edition, 1979.

[2] I. P. Castro and P. Bradshaw, "The turbulence structure of a highly curved mixing layer," Journal of Fluid Mechanics, vol. 73, no. 2, pp. 265-304, 1976.

[3] M. M. Gibson and W. Rodi, "A reynolds-stress closure model of turbulence applied to the calculation of a highly curved mixing layer," Journal of Fluid Mechanics, vol. 103, pp. 161-182, 1981. 
[4] R. D. Moser, M. M. Rogers, and D. W. Ewing, "Self-similarity of time-evolving plane wakes," Journal of Fluid Mechanics, vol. 367, pp. 255-289, 1998.

[5] M. M. Rogers, "The evolution of strained turbulent plane wakes," Journal of Fluid Mechanics, vol. 463, pp. 53-120, 2002.

[6] X. Wu and P. A. Durbin, "Evidence of longitudinal vortices evolved from distorted wakes in a turbine passage," Journal of Fluid Mechanics, vol. 446, pp. 199-228, 2001.

[7] P. Stadtmüller, "Investigation of Wake-Induced Transition on the LP turbine Cascade T106A-EIZ," DFG-VerbundprojectFo 136/11, Version 1.1, 2001.

[8] P. Stadtmüller and L. Fottner, "A test case for the numerical investigation of wake passing effects of a highly loaded LP turbine cascade blade," ASME Paper 2001-GT-311, 2001.

[9] J. G. Wissink, "DNS of a separating low Reynolds number flow in a turbine cascade with incoming wakes," in Proceedings of the 5th International Symposium on Engineering Turbulence Modelling and Measurements, Mallorca, Spain, September 2002.

[10] V. Michelassi, J. Wissink, and W. Rodi, "Analysis of DNS and LES of flow in a low pressure turbine cascade with incoming wakes and comparison with experiments," Flow, Turbulence and Combustion, vol. 69, no. 3-4, pp. 295-329, 2002.

[11] K. Hsu and S. L. Lee, "A numerical technique for two-dimensional grid generation with grid control at all of the boundaries," Journal of Computational Physics, vol. 96, no. 2, pp. 451-469, 1991.

[12] X. Wu, R. G. Jacobs, J. C. R. Hunt, and P. A. Durbin, "Simulation of boundary layer transition induced by periodically passing wakes," Journal of Fluid Mechanics, vol. 398, pp. 109-153, 1999.

[13] M. Breuer and W. Rodi, "Large eddy simulation for complex turbulent flows of practical interest," in Flow Simulation with HighPerformance Computers II, vol. 48 of Notes on Numerical Fluid Mechanics (NNFM), pp. 258-274, Vieweg+Teubner, Wiesbaden, 1996.

[14] M. Germano, U. Piomelli, P. Moin, and W. H. Cabot, "A dynamic subgrid-scale eddy viscosity model," Physics of Fluids A, vol. 3, no. 7, pp. 1760-1765, 1991.

[15] D. K. Lilly, "A proposed modification of the Germano subgridscale closure method," Physics of Fluids A, vol. 4, no. 3, pp. 633635, 1992.

[16] M. A. Leschziner and W. Rodi, "Calculation of annular and twin parallel jets using various discretization schemes and turbulence-model variations," Transaction of the ASME Journal of Fluids Engineering, vol. 103, no. 2, pp. 352-360, 1981.

[17] S. B. Pope, Turbulent Flows, Cambridge University Press, Cambridge, UK, 2001. 

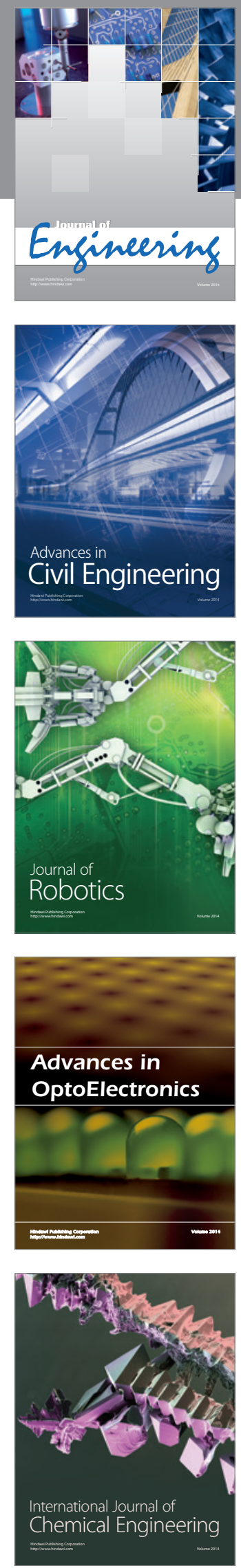

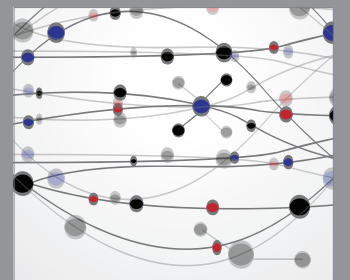

The Scientific World Journal
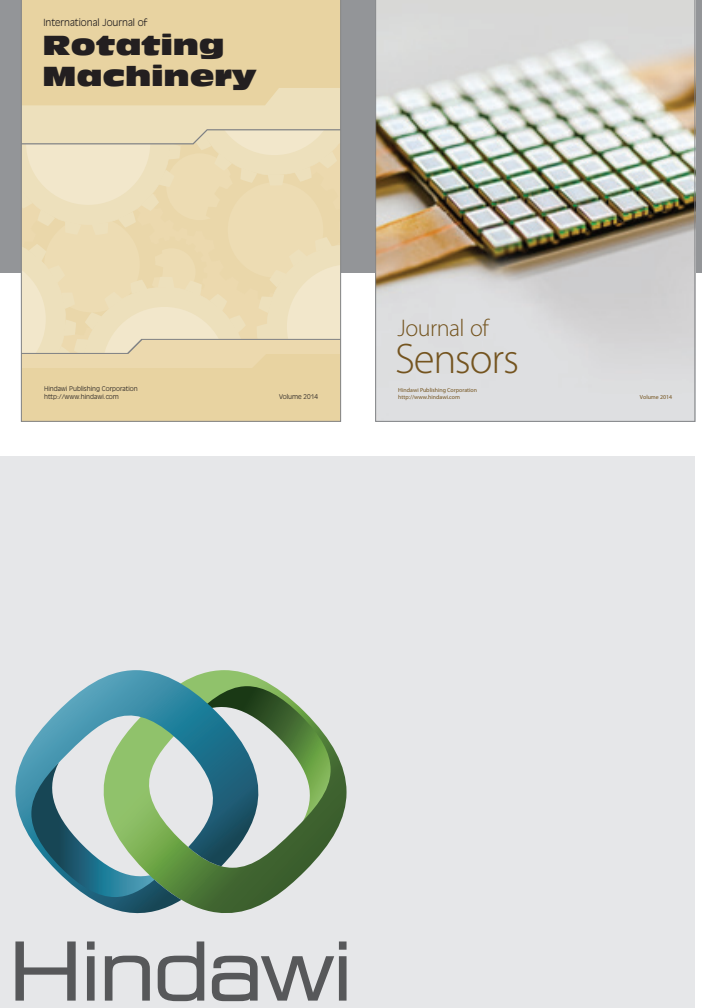

Submit your manuscripts at http://www.hindawi.com
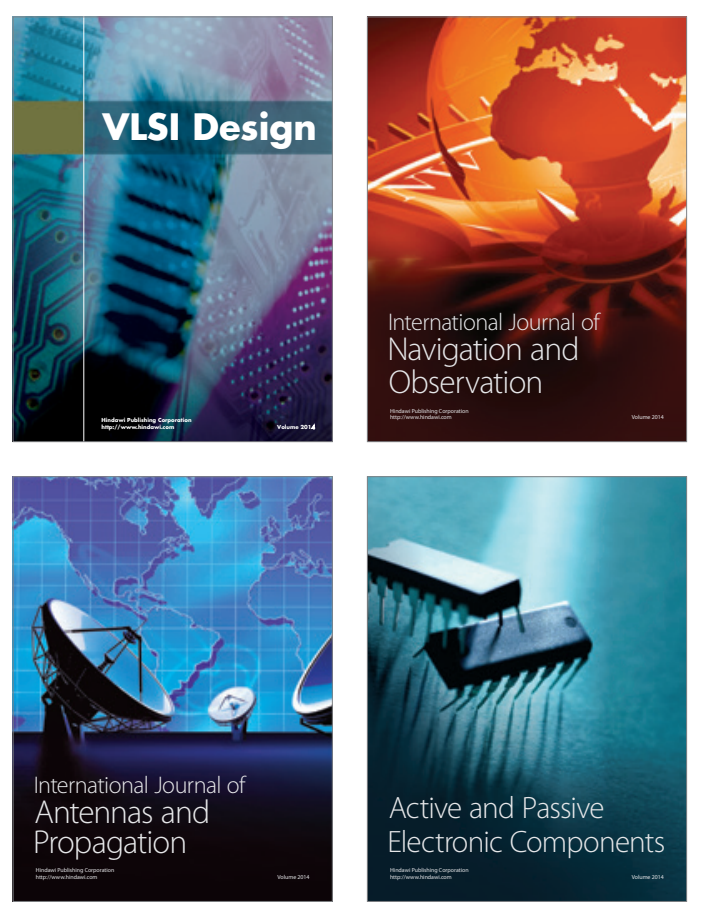
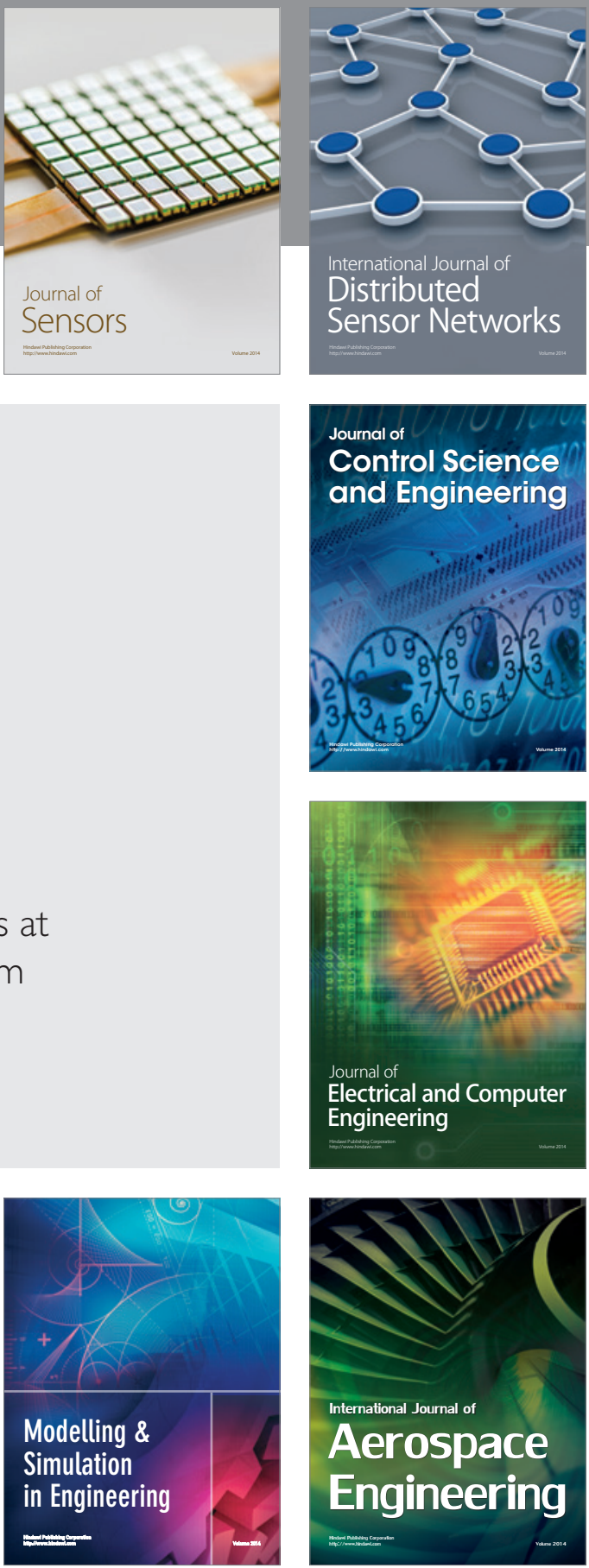

Journal of

Control Science

and Engineering
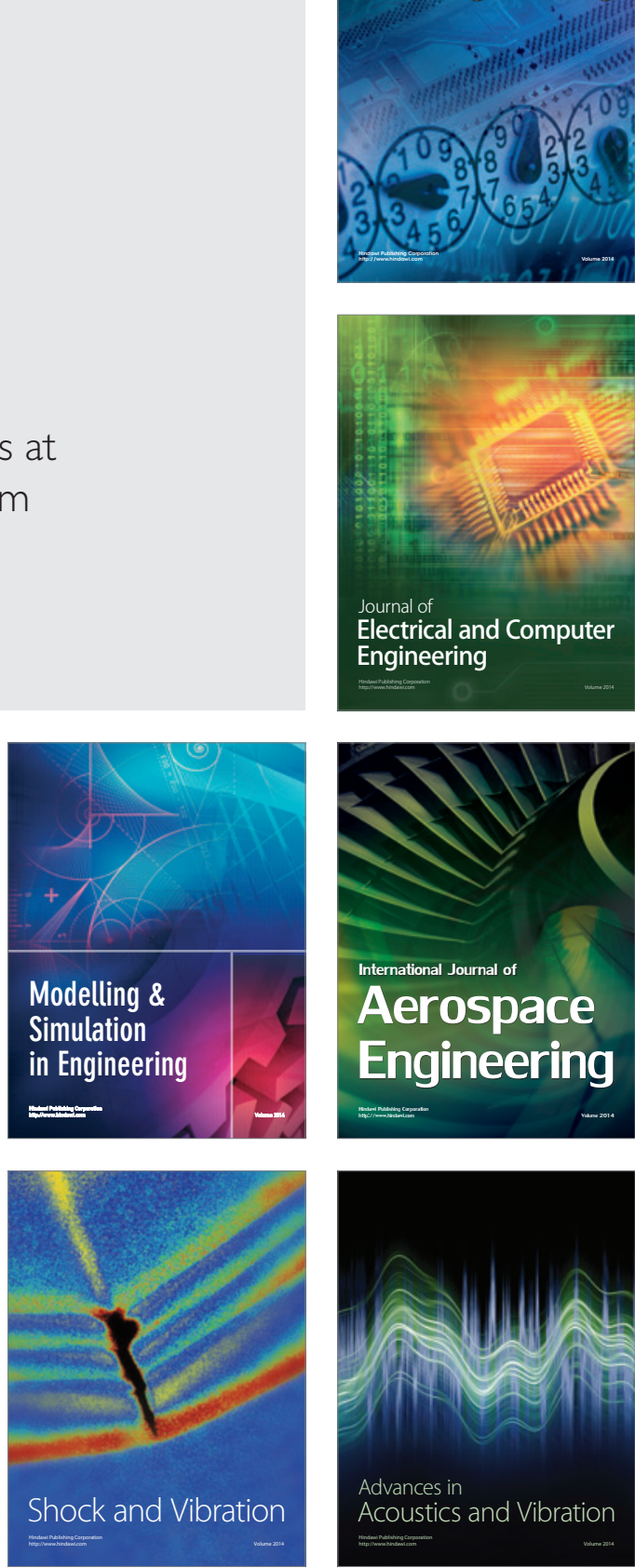